\title{
Bone graft options for spinal fusion following resection of spinal column tumors: systematic review and meta-analysis
}

\author{
*Benjamin D. Elder, MD, PhD, ${ }^{1}$ Wataru Ishida, MD, ${ }^{1}$ C. Rory Goodwin, MD, PhD, ${ }^{1}$ Ali Bydon, MD, ${ }^{1}$ \\ Ziya L. Gokaslan, MD, ${ }^{2}$ Daniel M. Sciubba, MD, ${ }^{1}$ Jean-Paul Wolinsky, MD, ${ }^{1}$ and \\ Timothy F. Witham, MD'
}

1'Department of Neurosurgery, The Johns Hopkins University School of Medicine, Baltimore, Maryland; and 2Department of Neurosurgery, Brown University School of Medicine, Providence, Rhode Island

\begin{abstract}
OBJECTIVE With the advent of new adjunctive therapy, the overall survival of patients harboring spinal column tumors has improved. However, there is limited knowledge regarding the optimal bone graft options following resection of spinal column tumors, due to their relative rarity and because fusion outcomes in this cohort are affected by various factors, such as radiation therapy (RT) and chemotherapy. Furthermore, bone graft options are often limited following tumor resection because the use of local bone grafts and bone morphogenetic proteins (BMPs) are usually avoided in light of microscopic infiltration of tumors into local bone and potential carcinogenicity of BMP. The objective of this study was to review and meta-analyze the relevant clinical literature to provide further clinical insight regarding bone graft options.
\end{abstract}

METHODS A web-based MEDLINE search was conducted in accordance with preferred reporting items for systematic review and meta-analysis (PRISMA) guidelines, which yielded 27 articles with 383 patients. Information on baseline characteristics, tumor histology, adjunctive treatments, reconstruction methods, bone graft options, fusion rates, and time to fusion were collected. Pooled fusion rates (PFRs) and $l^{2}$ values were calculated in meta-analysis. Meta-regression analyses were also performed if each variable appeared to affect fusion outcomes. Furthermore, data on 272 individual patients were available, which were additionally reviewed and statistically analyzed.

RESULTS Overall, fusion rates varied widely from $36.0 \%$ to $100.0 \%$ due to both inter- and intrastudy heterogeneity, with a PFR of $85.7 \%\left(1^{2}=36.4\right)$. The studies in which cages were filled with morselized iliac crest autogenic bone graft (ICABG) and/or other bone graft options were used for anterior fusion showed a significantly higher PFR of 92.8, compared with the other studies $(83.3 \%, p=0.04)$. In per-patient analysis, anterior plus posterior fusion resulted in a higher fusion rate than anterior fusion only $(98.8 \%$ vs $86.4 \%, p<0.001)$. Although unmodifiable, RT $(90.3 \%$ vs $98.6 \%, p=0.03)$ and lumbosacral tumors (74.6\% vs $97.9 \%, p<0.001$ ) were associated with lower fusion rates in univariate analysis. The mean time to fusion was $5.4 \pm 1.4$ months (range 3-9 months), whereas 16 of 272 patients died before the confirmation of solid fusion with a mean survival of $3.1 \pm 2.1$ months (range $0.5-6$ months). The average time to fusion of patients who received $R T$ and chemotherapy were significantly longer than those who did not receive these adjunctive treatments (RT: 6.1 months vs 4.3 months, $p<0.001$; chemotherapy: 6.0 months vs 4.3 months, $p=0.02$ ).

CONCLUSIONS Due to inter- and intrastudy heterogeneity in patient, disease, fusion criteria, and treatment characteristics, the optimal surgical techniques and factors predictive of fusion remain unclear. Clearly, future prospective, randomized studies will be necessary to better understand the issues surrounding bone graft selection following resection of spinal column tumors.

https://thejns.org/doi/abs/10.3171/2016.8.FOCUS16112

KEY WORDS bone graft; spinal fusion; spinal column tumors; cage reconstruction; bone morphogenetic protein; radiation therapy; chemotherapy; spinal oncology

ABBREVIATIONS BMD = bone mineral density; BMP = bone morphogenetic protein; $\mathrm{Cl}$ = confidence interval; $\mathrm{HR}=$ hazard ratio; $\mathrm{ICABG}=$ iliac crest autogenic bone graft; $\mathrm{OR}=$ odds ratio; $\mathrm{PBT}$ = proton beam therapy; PFR = pooled fusion rate; PRISMA = preferred reporting items for systematic review and meta-analysis; $\mathrm{RT}=$ radiation therapy. SUBMITTED March 24, 2016. ACCEPTED August 11, 2016.

INCLUDE WHEN CITING DOI: 10.3171/2016.8.FOCUS16112.

* Drs. Elder and Ishida contributed equally to this work. 
$\mathrm{W}$ ITH the advent of new adjunctive therapies such as stereotactic body radiation therapy, proton beam therapy, and molecular-targeted therapy, the overall survival rate of patients with spinal column tumors undergoing resection has been improving over the last two decades. ${ }^{24,28,29,37}$ Also, due to novel reconstruction techniques, such as titanium mesh cages and expandable cages, and various bone graft and bone graft extender options, more complex reconstructions have become feasible for patients following spinal column tumor resection. Hence, not only overall survival but also other clinical outcomes such as fusion rates, perioperative complications, postoperative neurological deficits, and long-term pain outcomes need to be taken into account to optimize functional outcomes. A significant factor that can significantly alter the quality of life of patients is stability of the spinal column, which is dependent on solid fusion and/or instrumentation. ${ }^{18}$ This is particularly true if resection of spinal column tumors involves spondylectomy, sacrectomy, and/ or resection of the entire posterior elements.

A major concern related to fusion procedures in spinal oncology is the influence of perioperative radiation therapy (RT) and chemotherapy on bone mineral density (BMD) and fusion outcomes. For instance, Hobusch et al. ${ }^{33}$ retrospectively reviewed 127 long-term survivors of chondrosarcoma who underwent RT and/or chemotherapy and concluded that they appear to be at greater risk for having low BMD and suffering from fractures than the healthy population. Proton beam therapy (PBT), which is often used in the treatment of chordomas, is also associated with decreased BMD. ${ }^{3,43}$ Furthermore, Emery et al. ${ }^{25}$ demonstrated that 4 of 25 patients $(16 \%)$ who underwent RT for malignant spinal column tumors before spinal fusion developed pseudarthrosis, and the total average radiation dose was higher for the pseudarthrosis group (48.9 Gy) than the fusion group (35.1 Gy). Lastly, Narayan et al..$^{53}$ reported a significantly lower fusion rate in patients with spinal column tumors compared with those with degenerative disc disease and trauma (54\% vs $91 \%$ vs $96 \%$, respectively), which was attributed to "systemic malignancy, local irradiation, and long-term use of systemic steroids." It is interesting that as adjunctive RT and chemotherapy have prolonged life expectancy of patients with spinal column tumors, their detrimental effects on BMD and fusion outcomes have paradoxically become problematic.

Additionally, tumor histology can substantially affect the surgical decision-making process. For instance, patients with primary or benign spinal column tumors often experience prolonged survival, ${ }^{17}$ which warrants the need for arthrodesis, although complications such as kyphosis, device subsidence, device loosening, or device fracture may be encountered..$^{2,4,15,46}$ In contrast, because patients harboring metastatic spinal column tumors or malignant tumors not amenable to en bloc resection may have relatively limited life expectancy, depending on factors such as tumor histology, extraspinal metastases, and performance status, ${ }^{64}$ achievement of fusion may not be required. ${ }^{21,54}$

Lastly, there are some limitations in bone graft options in spinal oncology. In general, the gold standard has been an iliac crest autogenic bone graft (ICABG), although it is associated with donor-site morbidity, ${ }^{12}$ as well as a higher pseudarthrosis rate in the setting of spinal oncology. ${ }^{30} \mathrm{Al}-$ ternatively, various structural and nonstructural autograft and allograft options as well as various bone graft extenders and enhancers are available. However, the use of local bone grafts such as laminae, facets, pedicles, and/or vertebrae is often avoided for patients with spinal column tumors due to potential microscopic invasion of tumor cells into adjacent areas, which limits the potential bone graft options. . $^{8,27,27,31,40}$ Additionally, although controversial, the application of bone morphogenetic protein-2 (BMP-2) to patients with tumors is generally contraindicated due to several prior studies that suggested an increased incidence of tumors in patients who received BMP-2..$^{58,62,63,66}$

Because of these issues, it is unclear what bone graft options should be selected in patients with spinal column tumors. The literature is also significantly limited, as a majority of prior reports were either retrospective with small numbers of patients,,${ }^{1,36}$ or case reports, ${ }^{5}$ focusing on survival outcomes or technical aspects of surgery rather than fusion outcomes. ${ }^{73}$ To the best of our knowledge, this is the first systematic review and meta-analysis focusing solely on bone graft options and fusion rates in the setting of spinal column tumor resection, aiming to provide some clinical insight into this controversial issue.

\section{Methods \\ Web-Based Literature Search}

A comprehensive web-based literature search was conducted utilizing Medline to collect all articles documenting bone graft options for spinal fusion following resection of spinal column tumors, complying with preferred reporting items for systematic review and meta-analysis (PRISMA) guidelines (Fig. 1). ${ }^{59}$ Using the following search terms, 2576 articles were identified from January 1, 1980, to February 1, 2016: "spine tumor" and "fusion" or "spinal tumor" and "fusion" or "spinal metastasis" and "fusion" or "spine metastasis" and "fusion" or "spine tumor" and "bone graft" or "spine tumor" and "autograft" or "spine tumor" and "allograft" or "spine tumor" and "BMP" or "spine tumor" and "bone morphogenetic protein" or "spine tumor" and "demineralized bone matrix" or "spine metastasis" and "autograft" or "spine metastasis" and "allograft" or "spine metastasis" and "BMP" or "spine metastasis" and "bone morphogenetic protein" or "spine metastasis" and "demineralized bone matrix" or "spine tumor" and "arthrodesis" or "spinal tumor" and "arthrodesis" or "spine tumor" and "reconstruction" or "spinal tumor" and "reconstruction" or "spine tumor" and "instrumentation" or "spinal tumor" and "instrumentation." Two independent authors reviewed each paper (B.D.E. and W.I.). If there were differences in opinions as to which articles should be included, a third reviewer was consulted (T.F.W.). Inclusion criteria were as follows: 1) full-texts were available, 2) articles were written in English, 3) studies were on human subjects, 4) more than 5 patients were included, and 5) fusion outcomes and bone graft used were reported. Technical case reports, review articles, ex vivo biomechanical studies, and articles not involving spinal column tumors were excluded. Following application of these inclusion and exclusion criteria, 27 relevant studies ${ }^{7,8,9,14,16,19,21,22,25,27,31,34,35,38-42,45,48,54,55,67,69-71,74}$ were 


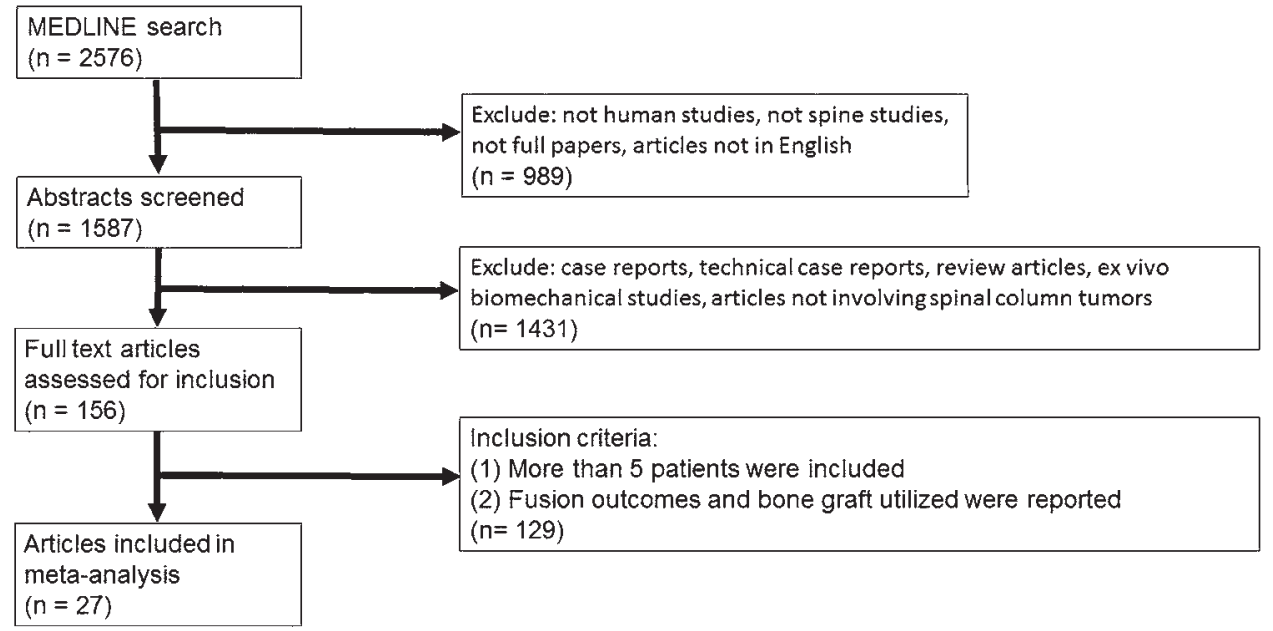

FIG. 1. Literature search results and screening process, based on the PRISMA guidelines.

identified. Clinical information on baseline characteristics, tumor histology, follow-up periods, mortality, adjunctive treatments, reconstruction methods, bone graft options, fusion assessment, fusion outcomes, and time to fusion were collected. Lastly, clinical data on 272 individual patients was available in 25 articles, $7-9,14,16,19,21,22,25,27,31,35,38-42,45,48,54,55$, ${ }^{67,69-71}$ which was further collected and analyzed.

\section{Statistical Analysis}

Statistical analyses were performed utilizing GraphPad Prism (version 6; GraphPad Software, Inc.) and Comprehensive Meta-Analysis Software (version 3, Biostat). Initially, a meta-analysis on 27 articles was conducted to calculate the overall pooled fusion rate. Next, the fusion rates, stratified by variables such as tumor histology, bone grafts, and reconstruction methods, were further analyzed. A fixed-effects model was applied in each analysis and the heterogeneity of the meta-analysis was calculated using $\mathrm{I}^{2}$ statistics. As previously described,$^{32} \mathrm{I}^{2}$ values less than $25 \%$ were considered as low heterogeneity, $25 \%-75 \%$ as moderate heterogeneity, and more than $75 \%$ as severe heterogeneity. Meta-regression analysis was additionally performed to clarify which variables affected the fusion rates and caused heterogeneity between studies; $p$ values, which tested the null hypothesis that the coefficient of each meta-regression analysis was equal to zero, were also calculated.

For analyses on individual patients' data, a Fisher's exact test was performed to compare intergroup differences stratified by categorical variables. Intergroup comparison of continuous variables was conducted using unpaired $t-$ tests. Data are presented as means \pm standard deviations unless noted otherwise. To identify factors related to pseudarthrosis, univariate analysis was initially performed and factors associated with pseudarthrosis in the univariate analysis $(\mathrm{p}<0.10)$ were subsequently entered into a multivariate logistic regression analysis. The odds ratios (ORs) for each variable were reported in addition to 95\% confidence intervals (CIs). For time-to-fusion analyses, Kaplan-Meier curves, stratified by variables, were drawn and compared between the two groups by the log-rank test. Hazard ratios (HRs) were calculated along with 95\% CIs. Finally, publication bias was assessed by drawing a funnel plot for the 27 control groups. The classic fail-safe $\mathrm{N}$ test was conducted and $\mathrm{p}$ values, which tested the null hypothesis that there was no publication bias and the funnel plot was symmetrical, were obtained. ${ }^{57}$ All reported $p$ values were two-sided and $p<0.05$ was considered to be statistically significant.

\section{Results \\ Overview of the Included Studies}

In 27 articles that met the inclusion criteria, fusion outcomes and bone graft materials were reported for 383 patients following resection of spinal column tumors. In Table 1, basic characteristics of each study are shown. The range of numbers of patients included in each study varied from 5 to 39. The evidence level was III in 2 articles, ${ }^{19,25}$ and IV in 25 studies. . $^{-9,14,16,21,22,27,31,34,35,38-42,45,48,54,55,67,69-71,74}$ The mean number of levels of tumor involvement ranged from 1 to 4.4 , and 5 studies $^{25,34,35,54,74}$ did not report this information. The number of articles reporting data on primary tumors, metastatic tumors, and both, were 9, 4, and 14, respectively. The mean follow-up periods of each study varied widely from 9 months to 119 months. At last follow-up, 0\%-29\% of patients harboring primary tumors had died, whereas mortality of patients with metastatic tumors ranged from $0 \%$ to $100 \%$. In Table 2, bone graft options and fusion outcomes are summarized. Thirteen studies $^{10,14,16,19,22,25,31,34,35,39,40,55,71}$ used bone grafts solely for anterior fusion, whereas two studies ${ }^{41,74}$ solely performed posterior fusion. The other 12 studies $7,8,21,27,38,42,45,48,54,67,69,70$ included patients who underwent either anterior fusion, posterior fusion, or both, and were thus substantially heterogeneous. Regarding graft options, 7 studies ${ }^{16,19,39,42,55,70,71}$ used cages filled with morselized bone graft or other bone graft extenders, whereas 4 studies, ${ }^{27,41,48,67}$ included patients with vascularized autogenic bone grafts either with or without vascular pedicles. Regarding adjunctive therapy, 18 studies, $7,8,14,16,21,22,25,35,38-42,45,48,67,70,74$ clearly stated the percentage of patients who underwent RT, which substan- 
TABLE 1. Summary of basic characteristics of each study

\begin{tabular}{|c|c|c|c|c|c|c|c|c|c|c|}
\hline $\begin{array}{l}\text { Authors } \\
\& \text { Year }\end{array}$ & $\begin{array}{c}\text { No. } \\
\text { of } \\
\text { Patients (\% } \\
\text { female) }\end{array}$ & $\begin{array}{l}\text { Mean } \\
\text { Age } \\
\text { in Yrs } \\
\text { (range) }\end{array}$ & $\begin{array}{c}\text { Mean } \\
\text { Levels } \\
\text { of Tumor } \\
\text { Involvement } \\
\text { (range) }\end{array}$ & Location & Tumor Histology & $\begin{array}{l}\text { Benign } \\
\text { or } \\
\text { Malignant }\end{array}$ & $\begin{array}{l}\text { Primary } \\
\text { or } \\
\text { Metastatic }\end{array}$ & $\begin{array}{l}\text { Mean } \\
\text { FU } \\
\text { in } \\
\text { Mos } \\
\text { (range) }\end{array}$ & $\begin{array}{l}\text { Mortality } \\
\text { Rate } \\
\text { at } \\
\text { Last FU } \\
(\%)\end{array}$ & $\begin{array}{l}1-Y r \\
\text { OS } \\
\text { Rate } \\
(\%)\end{array}$ \\
\hline $\begin{array}{r}\text { Bohlman et } \\
\text { al., } 1986\end{array}$ & $16(31)$ & $38(16-69)$ & 1 & C: 16 & $\begin{array}{l}\mathrm{CH} 4, \mathrm{HA} 2, \mathrm{OB} 1, \mathrm{OC} 1, \\
\mathrm{SP} 1, \mathrm{ISH} 1, \mathrm{MFH} 1, \\
\mathrm{OO} 1, \mathrm{SA} 1\end{array}$ & Mixed & Mixed & $63(1-216)$ & 44 & 88 \\
\hline $\begin{array}{l}\text { Emery et } \\
\text { al., } 1994\end{array}$ & $25(44)$ & NR & NR & $\begin{array}{l}\text { C: } 4 \\
\text { T: } 12 \\
\text { L: } 9\end{array}$ & $\begin{array}{c}\text { BC 6, PC 4, CH 2, LC 2, } \\
\text { MM 2, CC 1, HA 1, OSS } \\
\text { 1, RCC 1, others } 5\end{array}$ & Mixed & Mixed & $21(6-65)$ & 52 & NR \\
\hline $\begin{array}{l}\text { Jackson \& } \\
\text { Go- } \\
\text { kaslan, } \\
2000\end{array}$ & $13(39)$ & $45(26-74)$ & NR & LS: 13 & $\begin{array}{l}\text { CH 3, GCT 2, RCC 2, BC } \\
\text { 1, CC 1, CS 1, ME 1, } \\
\text { SCS 1, SEM } 1\end{array}$ & Mixed & Mixed & $20(3-50)$ & 31 & 77 \\
\hline Fidler, 2001 & $10(50)$ & $32(21-68)$ & $2.4(1-6)$ & $\begin{array}{l}\mathrm{T}: 9 \\
\mathrm{~L}: 1\end{array}$ & GCT & Benign & Primary & $89(30-144)$ & 10 & 100 \\
\hline $\begin{array}{l}\text { Coumans } \\
\text { et al., } \\
2002\end{array}$ & 15 (NR) & $59(31-81)$ & $1.7(1-3)$ & $\begin{array}{l}\text { C: } 12 \\
\text { T: } 3\end{array}$ & $\begin{array}{l}\text { LC 4, RCC 2, ISH 2, BC 1, } \\
\text { CC 1, MM 1, PA 1, PC } \\
\text { 1, SP 1, unknown } 1\end{array}$ & Mixed & Mixed & $9(0.5-15)$ & 27 & 70 \\
\hline $\begin{array}{l}\text { Boriani et } \\
\quad \text { al., } 2002\end{array}$ & $39(43)$ & $45(7-69)$ & $1.2(1-3)$ & $\begin{aligned} T: & 14 \\
L: & 28\end{aligned}$ & $\begin{array}{l}\text { Primary malignant tumor } \\
26 \text {, benign tumor } 7 \text {, soli- } \\
\text { tary metastatic tumor } 6 \text {, } \\
\text { plasmacytoma } 3\end{array}$ & Mixed & Mixed & $26(6-60)$ & 14 & NR \\
\hline $\begin{array}{l}\text { Heidecke } \\
\text { et al., } \\
2003\end{array}$ & $14(57)$ & $51(19-66)$ & $1.4(1-2)$ & C & $\begin{array}{l}\text { BC 6, MM 2, HS 1, LC 1, } \\
\text { ML 1, PC 1, RCC 1, } \\
\text { SEM } 1\end{array}$ & Malignant & Metastatic & $24(3-60)$ & 86 & 57 \\
\hline $\begin{array}{l}\text { Lewand- } \\
\text { rowski et } \\
\text { al., } 2004 \\
\end{array}$ & $30(60)$ & $47(17-76)$ & $1.3(1-5)$ & $\begin{aligned} T: & 14 \\
& L: 16\end{aligned}$ & $\begin{array}{l}\text { CS } 7, \text { OS } 6, \mathrm{CH} 4,3 \mathrm{RCC} \\
\text { 3, BC 2, CC 1, TC 1, } \\
\text { others } 6\end{array}$ & Mixed & Mixed & $14(7-60)$ & 100 & NR \\
\hline $\begin{array}{l}\text { Wilden et } \\
\quad \text { al., } 2006\end{array}$ & $13(62)$ & $44(18-73)$ & $4.4(1-10)$ & NR & $\begin{array}{l}\text { CS 3, PG 2, PT 2, ABC 1, } \\
\text { ES 1, mGCT 1, GN 1, } \\
\text { LES 1, RCC } 1\end{array}$ & Mixed & Mixed & $25(14-50)$ & 15 & 100 \\
\hline $\begin{array}{l}\text { Oda et al., } \\
2006\end{array}$ & 5 (unclear) & Unclear & NR & NR & Unclear & Malignant & Metastatic & 22 & 100 & NR \\
\hline $\begin{array}{l}\text { Denaro et } \\
\quad \text { al., } 2007\end{array}$ & $8(25)$ & $21(13-24)$ & 1 & $\begin{array}{l}\text { C-1: } 1 \\
\text { C-2: } 1 \\
\text { C: } 6\end{array}$ & OB & Benign & Primary & $39(32-44)$ & 0 & 100 \\
\hline $\begin{array}{l}\text { Barrene- } \\
\text { chea et } \\
\text { al., } 2007\end{array}$ & $7(43)$ & $34(6-61)$ & $2.9(2-4)$ & $\begin{array}{l}\text { C-2: } 1 \\
\text { C: } 6\end{array}$ & $\mathrm{CH}$ & Malignant & Primary & $59(7-163)$ & 29 & 86 \\
\hline $\begin{array}{l}\text { Liljenqvist } \\
\text { et al., } \\
2008\end{array}$ & 21 (NR) & $30(9-68)$ & $1.4(1-4)$ & $\begin{array}{l}\text { T: } 10 \\
\text { TL: } 1 \\
\text { L: } 10\end{array}$ & $\begin{array}{c}\text { ES } 8, \text { OSS } 6, \text { RCC } 3, \text { CS } \\
\text { 1, DF 1, MHF 1, PG } 1\end{array}$ & Malignant & Mixed & $41(8-114)$ & 24 & 90 \\
\hline $\begin{array}{l}\text { Junming et } \\
\text { al., } 2008\end{array}$ & $19(32)$ & $35(17-52)$ & $1.6(1-3)$ & $\begin{array}{l}\text { C-2: } 4 \\
\text { C: } 15\end{array}$ & GCT 19 & Benign & Primary & $67(36-124)$ & 16 & 100 \\
\hline $\begin{array}{l}\text { Chuang et } \\
\text { al., } 2008\end{array}$ & $17(41)$ & $55(34-77)$ & 1 & C & $\begin{array}{l}\mathrm{LC} 7, \mathrm{CC} 4, \mathrm{NAC} 2, \mathrm{BC} 2 \\
\quad \mathrm{HC} 1, \mathrm{CHC} 1\end{array}$ & Malignant & Metastatic & NR & NR & NR \\
\hline $\begin{array}{l}\text { Moran et } \\
\quad \text { al., } 2009\end{array}$ & $7(29)$ & $30(14-55)$ & $3.3(2-5)$ & $\begin{array}{l}\text { LS: } 4 \\
\text { SAC: } \\
3\end{array}$ & $\begin{array}{l}\text { CS 2, ES 1, ME 1, mGCT } \\
\text { 1, OSS 1, SCS } 1\end{array}$ & Malignant & Mixed & $45(19-98)$ & 14 & NR \\
\hline $\begin{array}{l}\text { Omeis et } \\
\quad \text { al., } 2010\end{array}$ & $5(60)$ & $67(58-77)$ & $2.6(2-4)$ & $\begin{array}{c}\text { C: } 3 \\
\text { CVT: } \\
2\end{array}$ & $\mathrm{BC} 2, \mathrm{CC} 2, \mathrm{LC} 1$ & Malignant & Metastatic & $13(1-27)$ & 0 & 100 \\
\hline
\end{tabular}


TABLE 1. Summary of basic characteristics of each study

\begin{tabular}{|c|c|c|c|c|c|c|c|c|c|c|}
\hline $\begin{array}{l}\text { Authors } \\
\& \text { Year }\end{array}$ & $\begin{array}{c}\text { No. } \\
\text { of } \\
\text { Patients (\% } \\
\text { female) }\end{array}$ & $\begin{array}{l}\text { Mean } \\
\text { Age } \\
\text { in Yrs } \\
\text { (range) }\end{array}$ & $\begin{array}{c}\text { Mean } \\
\text { Levels } \\
\text { of Tumor } \\
\text { Involvement } \\
\text { (range) }\end{array}$ & Location & Tumor Histology & $\begin{array}{l}\text { Benign } \\
\text { or } \\
\text { Malignant }\end{array}$ & $\begin{array}{l}\text { Primary } \\
\text { or } \\
\text { Metastatic }\end{array}$ & $\begin{array}{l}\text { Mean } \\
\text { FU } \\
\text { in } \\
\text { Mos } \\
\text { (range) }\end{array}$ & $\begin{array}{c}\text { Mortality } \\
\text { Rate } \\
\text { at } \\
\text { Last FU } \\
(\%)\end{array}$ & $\begin{array}{c}1-Y r \\
\text { OS } \\
\text { Rate } \\
(\%)\end{array}$ \\
\hline $\begin{array}{l}\text { Hu et al., } \\
2010\end{array}$ & $18(33)$ & $48(34-65)$ & NR & TL or $\mathrm{L}$ & $\begin{array}{l}\text { GCT 2, SP 2, CS 1, ES } \\
\text { 1, BC 2, LC 2, PC 2, } \\
\text { RCC 2, LES 1, TC 1, } \\
\text { unknown 2 }\end{array}$ & Mixed & Mixed & $24(18-36)$ & 17 & 83 \\
\hline $\begin{array}{l}\text { Yang et al., } \\
2011^{70}\end{array}$ & $11(36)$ & $50(23-77)$ & $1.4(1-3)$ & $\mathrm{C}-2$ & $\begin{array}{l}\mathrm{CH} \text { 2, GCT 2, SP 2, EG } \\
\text { 1, HP 1, LC 1, NAC 1, } \\
\text { PC } 1\end{array}$ & Mixed & Mixed & $21(12-37)$ & 27 & 90 \\
\hline $\begin{array}{l}\text { Yang et al., } \\
2011^{69}\end{array}$ & $9(56)$ & $39(19-61)$ & $1.2(1-2)$ & C-1 & $\begin{array}{l}\text { OC 3, OB 2, CS 1, EG 1, } \\
\text { GCT 1, SP } 1\end{array}$ & Mixed & Primary & $53(16-100)$ & 11 & 100 \\
\hline $\begin{array}{l}\text { Kawahara } \\
\text { et al., } \\
2011\end{array}$ & $10(50)$ & $42(16-59)$ & $1.6(1-3)$ & L & $\begin{array}{l}\text { GCT 4, BC 2, ABC 1, HA } \\
\text { 1, LPS, RCC } 1\end{array}$ & Mixed & Mixed & $52(14-124)$ & 20 & 100 \\
\hline $\begin{array}{l}\text { Lewis et al., } \\
\quad 2012\end{array}$ & $17(29)$ & $51(16-74)$ & $2.1(1-3)$ & $\mathrm{T}: 17$ & LC $10, \operatorname{CS} 3,003$, OSS 1 & Mixed & Mixed & $28(2-72)$ & 35 & 65 \\
\hline $\begin{array}{c}\text { Yoshioka et } \\
\text { al., } 2013\end{array}$ & $22(59)$ & $33(52-68)$ & $3.3(3-5)$ & $\begin{array}{r}\text { CVT: } 2 \\
\text { T: } 15 \\
\text { TL: } 5\end{array}$ & $\begin{array}{l}\text { RCC 7, BC 3, GCT 3, TC } \\
\text { 3, AS 1, CH 1, CS 1, LC } \\
\text { 1, LES 1, SM } 1\end{array}$ & Mixed & Mixed & $26(12-67)$ & 14 & 100 \\
\hline $\begin{array}{l}\text { Zheng et } \\
\text { al., } 2014\end{array}$ & $6(17)$ & $31.8(18-52)$ & NR & CVJ & EG & Benign & Primary & $77(37-140)$ & 0 & 100 \\
\hline $\begin{array}{l}\text { Menezes et } \\
\text { al., } 2014\end{array}$ & $13(31)$ & $11(4-17)$ & $1.3(1-2)$ & CVJ & $\begin{array}{l}\mathrm{CH} 4, \mathrm{FD} 3, \mathrm{ABC} 2, \mathrm{OB} 2 \\
\quad \mathrm{EG} 1, \mathrm{OC} 1\end{array}$ & Mixed & Primary & $119(24-204)$ & 0 & 100 \\
\hline $\begin{array}{l}\text { Domovitov } \\
\text { et al., } \\
2016\end{array}$ & $8(63)$ & $33(25-55)$ & $2.4(1-4)$ & SAC & GCT & Benign & Primary & $99(2-288)$ & 25 & 88 \\
\hline $\begin{array}{l}\text { Chen et al., } \\
2015\end{array}$ & $5(60)$ & $31(23-45)$ & 1 & C & GCT & Benign & Primary & $32(10-48)$ & 0 & 100 \\
\hline
\end{tabular}

$\mathrm{ABC}=$ aneurysmal bone cyst; $\mathrm{AS}=$ angiosarcoma; $\mathrm{BC}=$ breast cancer $\mathrm{C}=$ cervical; $\mathrm{CC}=$ colon cancer; $\mathrm{CH}=$ chordoma; $\mathrm{CHC}=$ cholangiocarcinoma; $\mathrm{CS}=$ chondrosarcoma; CVJ = craniovertebral junction; CVT = cervicothoracic; DF = desmoplastic fibroma; EG = eosinophilic granuloma; ES = epithelioid sarcoma; FD = fibrous dysplasia; FU = follow-up; GCT = giant cell tumor; GN = ganglionic neuroblastoma; HA = hemangioma; HC = hepatic cancer; $\mathrm{HP}=$ hemangioperithelioma; HS = histiocytoma; ISH = intraosseous schwannoma; L = lumbar; LC = lung cancer; LES = leiomyosarcoma; LPS = liposarcoma; LS = lumbosacral; ME = myxopapillary ependymoma; $\mathrm{MFH}=$ malignant fibrous histiocytoma; $\mathrm{ML}=$ melanoma; $\mathrm{MM}=$ multiple myeloma; $\mathrm{mGCT}=$ malignant $\mathrm{GCT}$; NAC = nasopharyngeal cancer; $\mathrm{NR}=$ not reported, $\mathrm{OB}=$ osteoblastoma; $\mathrm{OC}=$ osteochondroma; $\mathrm{OO}=$ osteoid osteoma; $\mathrm{OS}=$ overall survival; OSS = osteosarcoma; $\mathrm{PA}=$ parotid adenocarcinoma; $\mathrm{PC}=$ prostate cancer; $\mathrm{PG}=$ paraganglioma; $\mathrm{PT}=$ pancoast tumor; $\mathrm{RCC}=$ renal cell carcinoma; $\mathrm{SA}=$ other sarcomas; $\mathrm{SAC}=$ sacral; $\mathrm{SCS}=$ spindle cell sarcoma; $\mathrm{SEM}=$ seminoma; $\mathrm{SM}=$ "Salida of macilla"; SP = solitary plasmacytoma; $\mathrm{T}=$ thoracic; $\mathrm{TC}=$ thyroid cancer; $\mathrm{TL}=$ thoracolumbar.

All studies were single-center, retrospective study designs, except for Emery et al., 1994 (multicenter, retrospective), and Coumans et al., 2002 (single-center, prospective). The quality of each study was Level IV, except for Coumans et al., 2002, and Emery et al., 1994 (both Level III).

tially varied from $0 \%$ to $100 \%$, whereas 14 studies $^{7,8,21,22,}$ $25,34,35,39,41,42,45,67,70,74$ provided the percentage of those who underwent chemotherapy, which ranged from $0 \%$ to $59 \%$. Information on preoperative embolization was available in

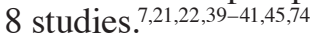

\section{Meta-Analysis and Meta-Regression Analysis}

Overall, fusion rates varied widely from $36.0 \%$ to $100.0 \%$, with a pooled fusion rate (PFR) of $85.7 \%(95 \%$ CI $80.6 \%-89.6 \%)$. The $\mathrm{I}^{2}$ value was 36.4 , which suggested moderate heterogeneity (Fig. 2). Following stratification into primary tumors and metastatic tumors, the PFRs and $\mathrm{I}^{2}$ values were $91.9 \%$ and 0 for primary tumors, and $79.5 \%$ and 58.3 for metastatic tumors, respectively $(\mathrm{p}=0.21$; Fig. $3)$. The studies in which cages were used for anterior fu- sion showed a PFR of 92.8 and $\mathrm{I}^{2}$ value of 0 , whereas those without use of cages demonstrated a PFR of $83.3 \%$ and an $\mathrm{I}^{2}$ value of 47.1 ( $\mathrm{p}=0.04$; Fig. 4). The comparison between vascularized bone grafts and others revealed that the PFR was higher in the vascularized bone graft group (93.0\% vs $84.8 \%$ ), although this difference was not statistically significant in the meta-regression analysis ( $p=0.21$; Fig. 5). Lastly, the PFRs and the $\mathrm{I}^{2}$ values for the articles whose primary outcomes were fusion outcomes versus others were $81.7 \%$ and 55.1 versus $91.6 \%$ and 0 , respectively ( $\mathrm{p}$ $=0.04$; Fig. 6).

\section{Analysis on Individual Patient Data}

For variables difficult to assess in the meta-analyses above due to intrastudy heterogeneity, analyses on avail- 


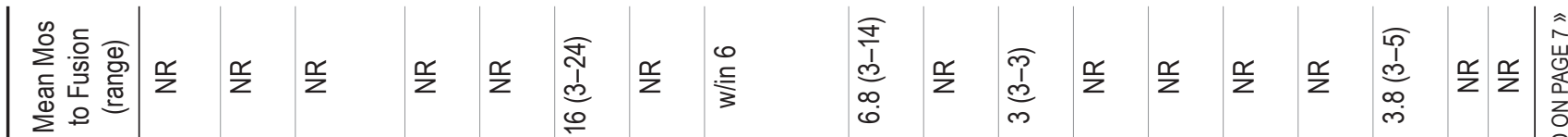

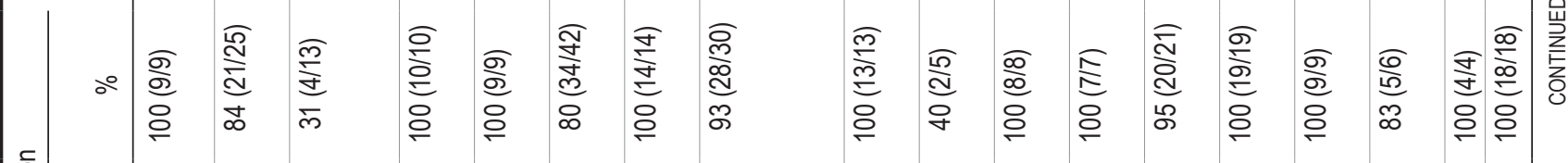

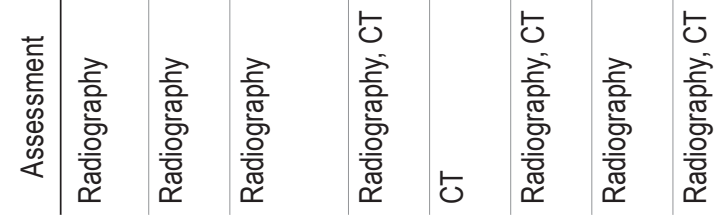
$\begin{array}{lllllll}\longleftarrow & \ddots & \ddots & \ddots & \ddots & \ddots & \ddots\end{array}$

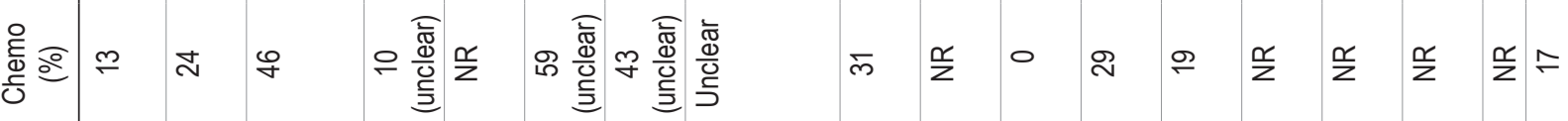

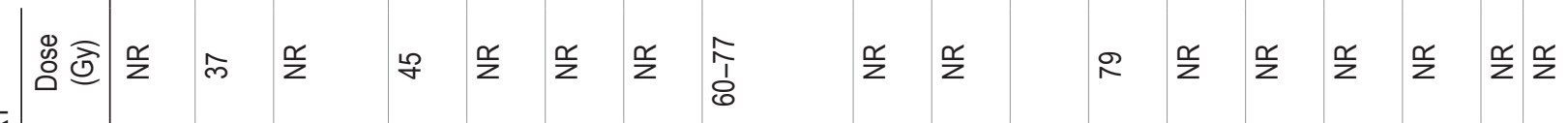
땀

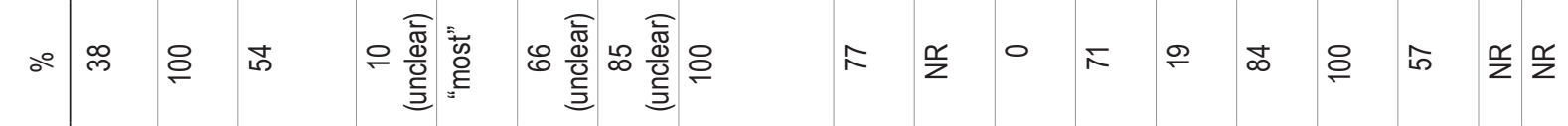

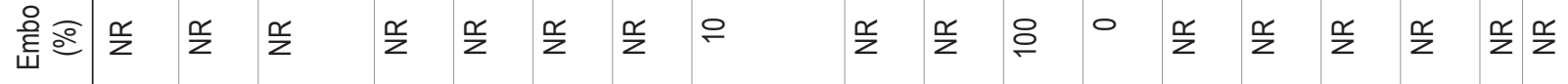

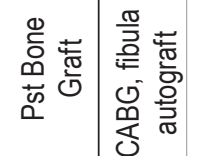

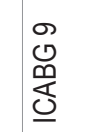
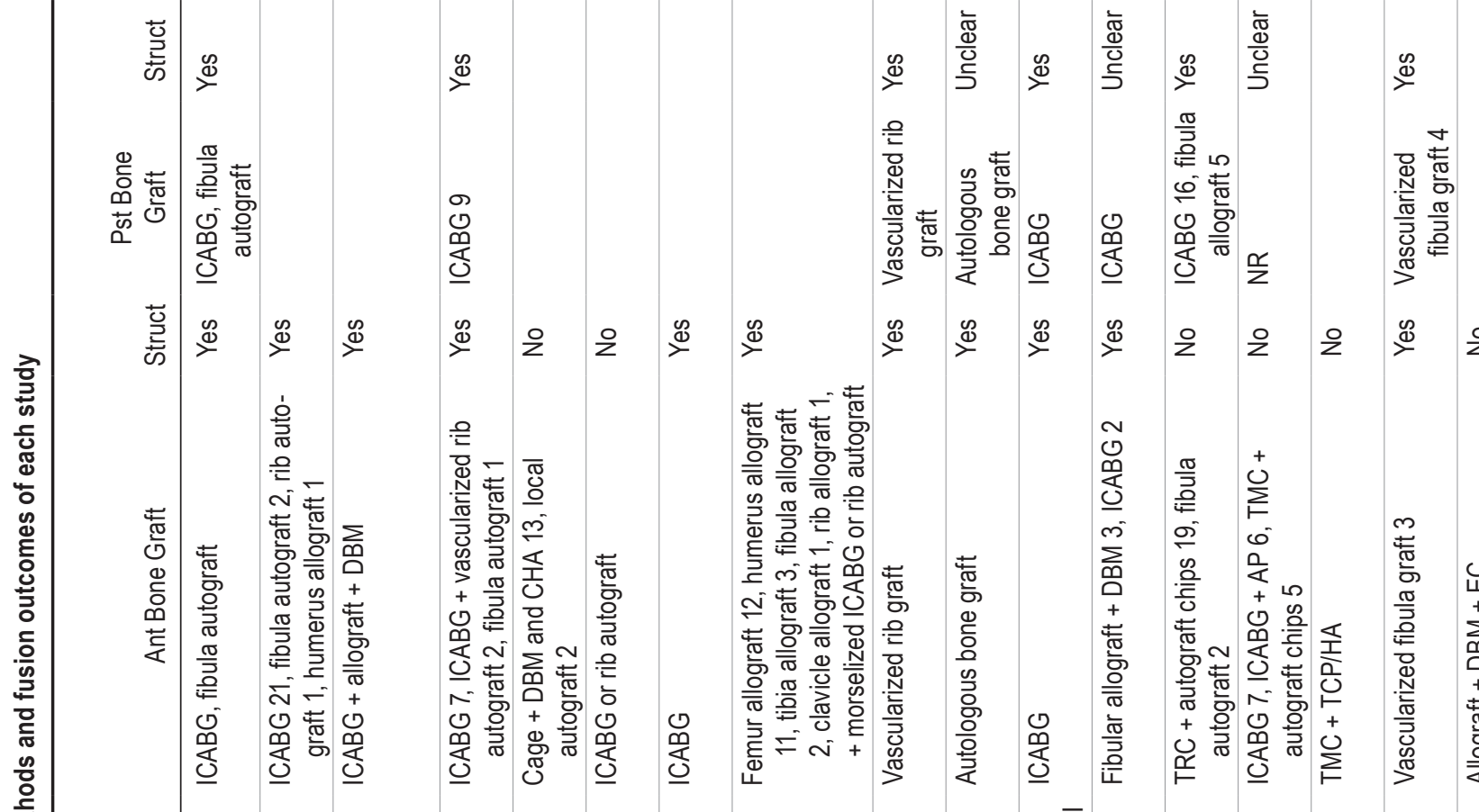

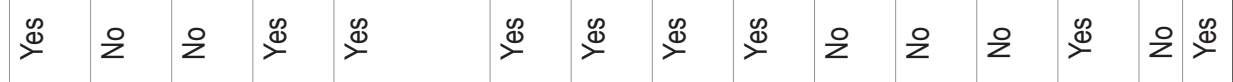
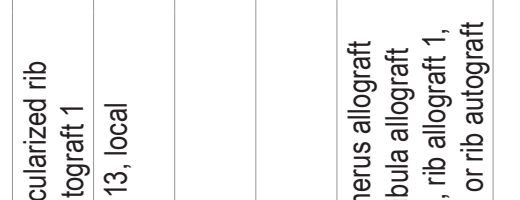

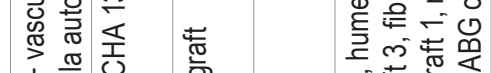

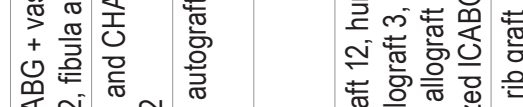

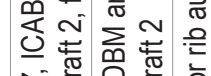

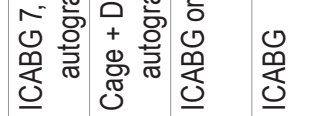

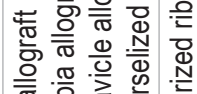

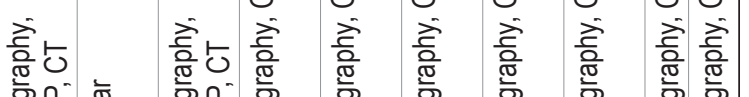

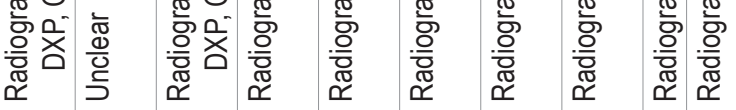

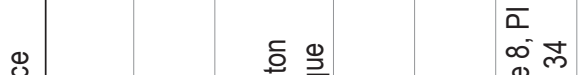

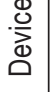

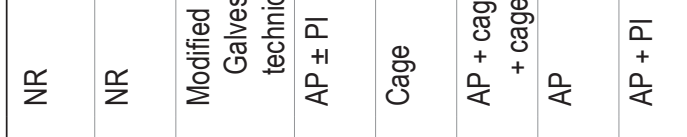

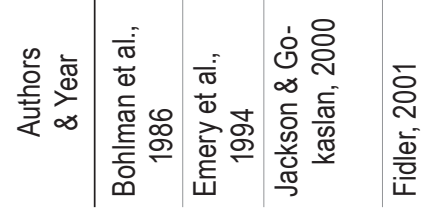
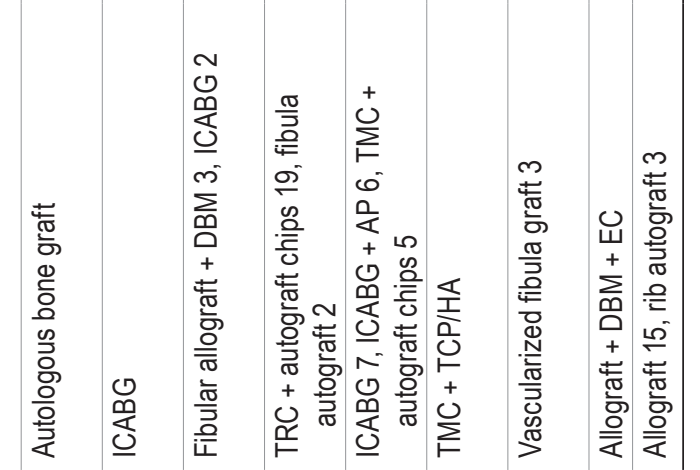

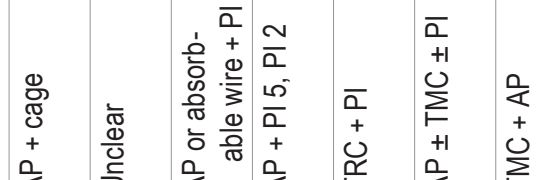

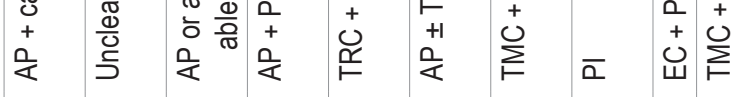

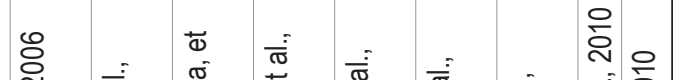
ㅎํำ

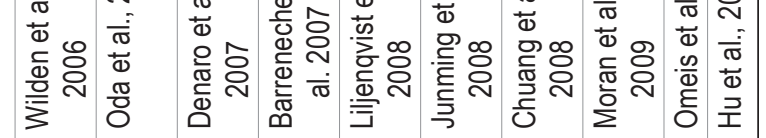




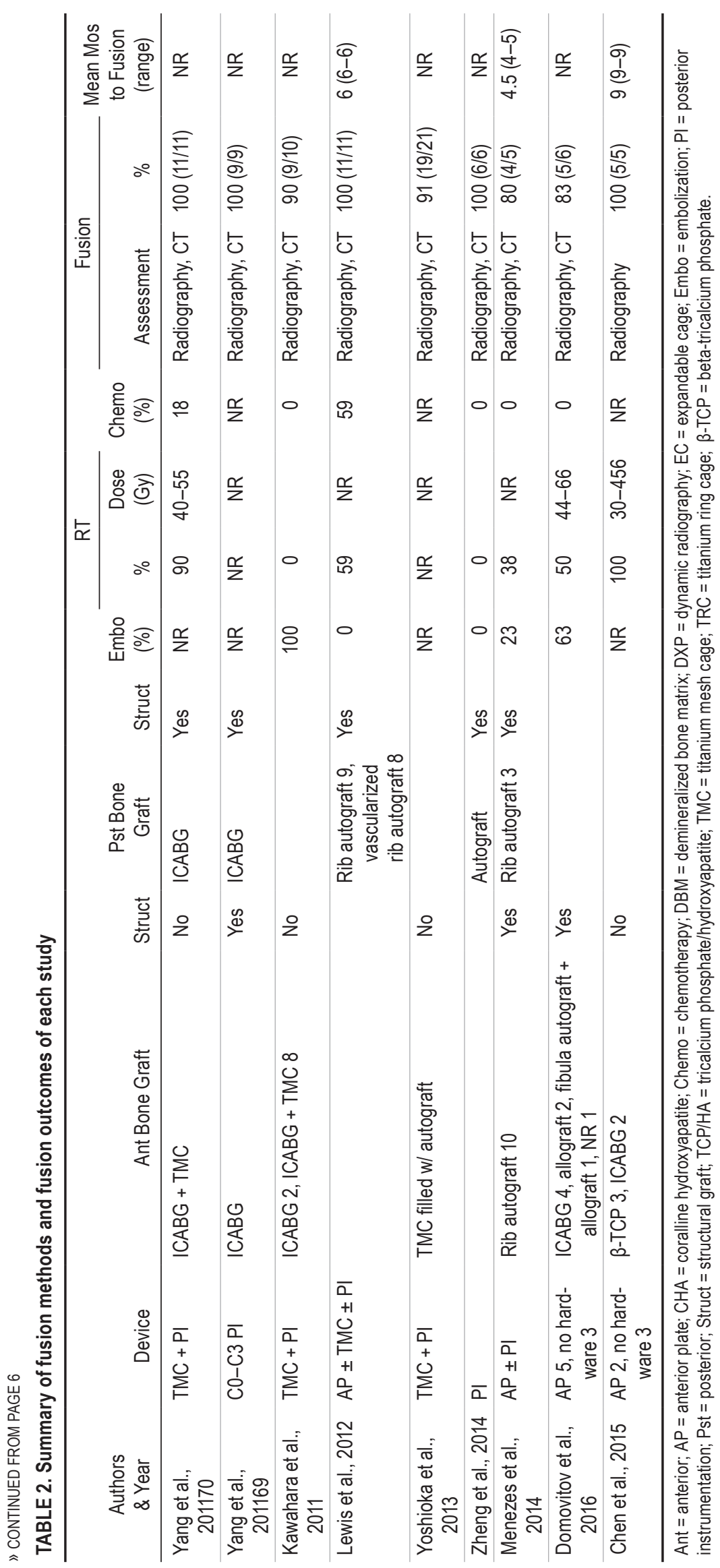




\begin{tabular}{|c|c|c|c|c|c|c|}
\hline & \multicolumn{5}{|c|}{ Statistics for each study } \\
\hline \multicolumn{2}{|l|}{ Study name } & $\begin{array}{c}\text { Event } \\
\text { rate }\end{array}$ & $\begin{array}{l}\text { Lower } \\
\text { limit }\end{array}$ & $\begin{array}{c}\text { Upper } \\
\text { limit }\end{array}$ & z-Value & p-Value \\
\hline Bohlman et al. & 1986 & 0.950 & 0.525 & .997 & 2.029 & 0.042 \\
\hline Emery et al. & 1994 & 0.840 & 0.643 & 0.939 & 3.040 & 0.002 \\
\hline Jackson et a & 2000 & 0.308 & 0.120 & 0.591 & -1.349 & 0.177 \\
\hline Filder et al. & 2001 & 0.955 & 0.552 & 0.997 & 2.103 & 0.035 \\
\hline Coumans et al. 2 & 2002 & 0.950 & 0.525 & 0.997 & 2.029 & 0.042 \\
\hline Boriani et al. & 2002 & 0.810 & 0.663 & 0.902 & 3.682 & 0.000 \\
\hline Heidecke et al. & 2003 & 0.967 & 0.634 & 0.998 & 2.341 & 0.019 \\
\hline \multicolumn{2}{|c|}{ Lewandrowski et al. 2004} & 0.933 & 0.769 & 0.983 & 3.606 & 0.000 \\
\hline Wilden et al. & 2006 & 0.964 & 0.616 & 0.998 & 2.289 & 0.022 \\
\hline Oda et al. & 2006 & 0.400 & 0.100 & 0.800 & -0.444 & 0.657 \\
\hline Denaro et al. & 2007 & 0.944 & 0.495 & 0.997 & 1.947 & 0.052 \\
\hline Barrenechea et al. & I. 2007 & 0.938 & 0.461 & 0.996 & 854 & 0.064 \\
\hline Liljenquist et al. & 2008 & 0.952 & 0.729 & 0.993 & 2.924 & 0.003 \\
\hline Junming et al. & 2008 & 0.975 & 0.702 & 0.998 & 2.558 & 0.011 \\
\hline Chuang et al. & 2008 & 0.950 & 0.525 & 0.997 & 2.029 & 0.042 \\
\hline Moran et al. & \multirow{2}{*}{$\begin{array}{l}2009 \\
2010\end{array}$} & 0.833 & 0.369 & 0.977 & 1.469 & 0.142 \\
\hline Omeis et al. & & 0.900 & 0.326 & 0.994 & 1.474 & 0.140 \\
\hline Hu et al. & 2010 & 0.974 & 0.690 & 0.998 & 2.519 & 0.012 \\
\hline \multicolumn{2}{|c|}{ Yang et al. (Neurosurgery) $2011^{70}$} & 0.958 & 0.575 & 0.997 & 2.170 & 0.030 \\
\hline Yang et al. (Spine) & osurgery) 2011 & 0.950 & 0.525 & 0.997 & 2.029 & 0.042 \\
\hline Kawahara et al. & 2011 & 0.900 & 0.533 & 0.986 & 2.084 & 0.037 \\
\hline Lewis et al. & 2012 & 0.958 & 0.575 & 0.997 & 2.170 & 0.030 \\
\hline Yoshioka et al. & 2013 & 0.905 & 0.689 & 0.976 & 3.028 & 0.002 \\
\hline Zheng et al. & 2014 & 0.929 & 0.423 & 0.996 & 1.748 & 0.081 \\
\hline Menezes et al. & 2014 & 0.800 & 0.309 & 0.973 & 1.240 & 0.215 \\
\hline Domovitov et al. & 2016 & 0.833 & 0.369 & 0.977 & 1.469 & 0.142 \\
\hline Chen et al. & 2015 & 0.917 & 0.378 & 0.995 & 1.623 & 0.105 \\
\hline & & 0.857 & 0.806 & 0.896 & 9.690 & 0.000 \\
\hline
\end{tabular}

$$
1^{2}=36.4
$$

Statistics for each study

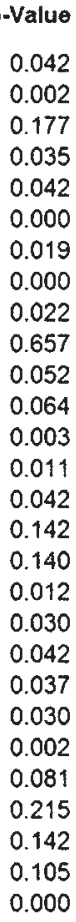

FIG. 2. Fusion rates of each study and the result of the meta-analysis.
Event rate and $95 \% \mathrm{Cl}$

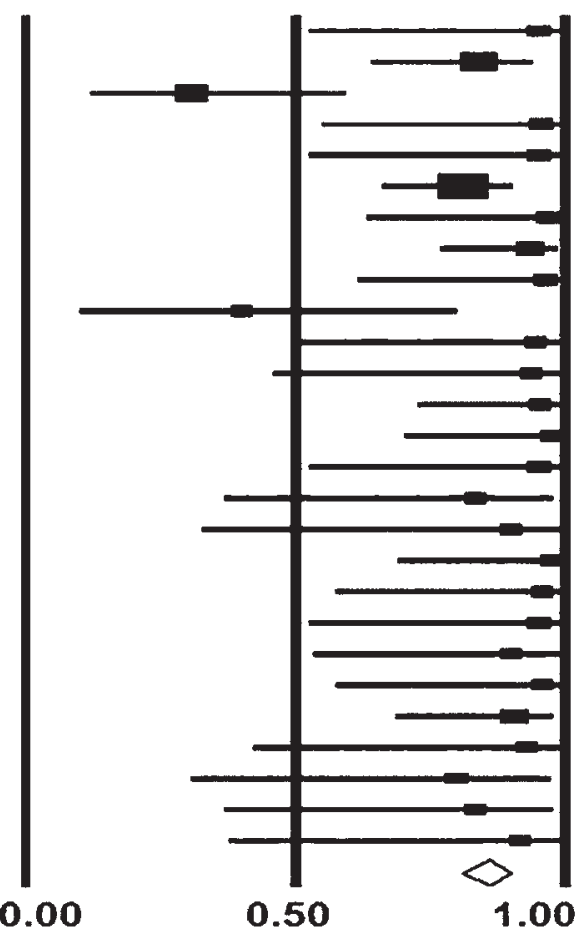

able individual patient data were further performed. The fusion rates stratified by each variable are shown in Table 3. In univariate analyses, tumors at junctional locations had a significantly lower fusion rate of $84.5 \%$ as opposed to $94.1 \%$ for nonjunctional tumors $(\mathrm{p}=0.02)$. Furthermore, tumors in lumbar and/or sacral regions demonstrated a significantly lower fusion rate of $74.6 \%$ compared with other regions of the spine $(97.9 \%, \mathrm{p}<0.001)$. In terms of adjunctive treatments, perioperative radiation was associated with lower fusion rates $(90.3 \%$ vs $98.6 \%, \mathrm{p}=0.03)$. Perioperative chemotherapy and preoperative embolization were not related to fusion status with statistical significance. For analyses on reconstruction techniques, the combined anterior and posterior fusion group resulted in a higher fusion

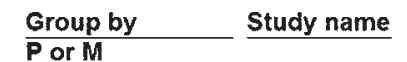

\begin{tabular}{|c|c|c|}
\hline \multirow{9}{*}{$\begin{array}{c}\text { Primary } \\
1^{2}=0\end{array}$} & Filder et al. & 2001 \\
\hline & Denaro et al. & 2007 \\
\hline & Barrenechea et a & 1. 2007 \\
\hline & Junming et al. & 2008 \\
\hline & Yang et al. (Spine & e) $2011^{69}$ \\
\hline & Zheng et al. & 2014 \\
\hline & Menezes et al. & 2014 \\
\hline & Domovitov et al. & 2016 \\
\hline & Chen et al. & 2015 \\
\hline \multirow{4}{*}{$\begin{array}{c}\text { Metastasis } \\
\mathrm{I}^{2}=58.3\end{array}$} & Heidecke et al. & 2003 \\
\hline & Oda et al. & 2006 \\
\hline & Chuang et al. & 2008 \\
\hline & Omeis et al. & 2010 \\
\hline \multicolumn{3}{|c|}{$\begin{array}{l}\text { Meta-regression } \\
\qquad p=0.21\end{array}$} \\
\hline
\end{tabular}

Statistics for each study

Event Lower Upper

rate limit limit $z$-Value $p$-Value

$\begin{array}{lllll}0.955 & 0.552 & 0.997 & 2.103 & 0.035 \\ 0.944 & 0.495 & 0.997 & 1.947 & 0.052 \\ 0.938 & 0.461 & 0.996 & 1.854 & 0.064 \\ 0.975 & 0.702 & 0.998 & 2.558 & 0.011 \\ 0.950 & 0.525 & 0.997 & 2.029 & 0.042 \\ 0.929 & 0.423 & 0.996 & 1.748 & 0.081 \\ 0.800 & 0.309 & 0.973 & 1.240 & 0.215 \\ 0.833 & 0.369 & 0.977 & 1.469 & 0.142 \\ 0.917 & 0.378 & 0.995 & 1.623 & 0.105 \\ 0.919 & 0.824 & 0.965 & 5.394 & 0.000 \\ 0.967 & 0.634 & 0.998 & 2.341 & 0.019 \\ 0.400 & 0.100 & 0.800 & -0.444 & 0.657 \\ 0.950 & 0.525 & 0.997 & 2.029 & 0.042 \\ 0.900 & 0.326 & 0.994 & 1.474 & 0.140 \\ 0.795 & 0.535 & 0.929 & 2.185 & 0.029 \\ 0.887 & 0.793 & 0.941 & 5.648 & 0.000\end{array}$

Event rate and $95 \% \mathrm{Cl}$

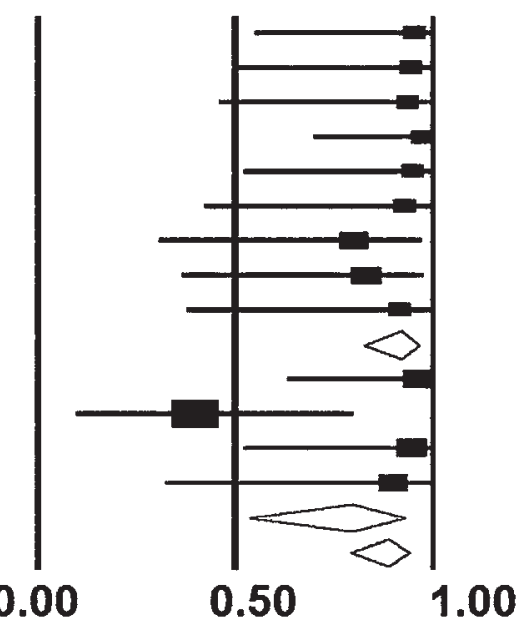

FIG. 3. The result of the meta-analysis and the meta-regression analysis, stratified by primary $(P)$ tumors versus metastatic (M) tumors. 


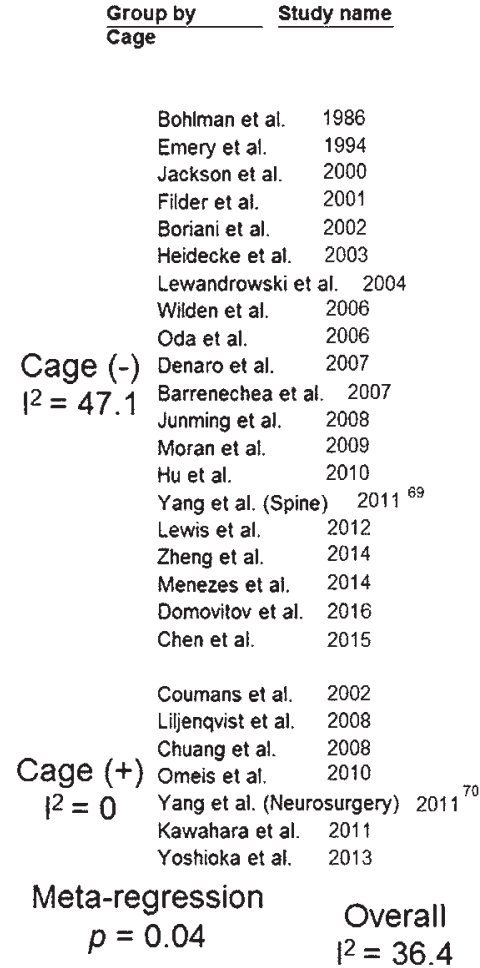

\begin{tabular}{ccccc}
\multicolumn{5}{c}{ Statistics for each study } \\
\cline { 2 - 4 } Event & Lower & Upper & & \\
rate & Ilmit & limit & z-Value & p-Value \\
0.950 & 0.525 & 0.997 & 2.029 & 0.042 \\
0.840 & 0.643 & 0.939 & 3.040 & 0.002 \\
0.308 & 0.120 & 0.591 & -1.349 & 0.177 \\
0.955 & 0.552 & 0.997 & 2.103 & 0.035 \\
0.810 & 0.663 & 0.902 & 3.682 & 0.000 \\
0.967 & 0.634 & 0.998 & 2.341 & 0.019 \\
0.933 & 0.769 & 0.983 & 3.606 & 0.000 \\
0.964 & 0.616 & 0.998 & 2.289 & 0.022 \\
0.400 & 0.100 & 0.800 & -0.444 & 0.657 \\
0.944 & 0.495 & 0.997 & 1.947 & 0.052 \\
0.938 & 0.461 & 0.996 & 1.854 & 0.064 \\
0.975 & 0.702 & 0.998 & 2.558 & 0.011 \\
0.833 & 0.369 & 0.977 & 1.469 & 0.142 \\
0.974 & 0.690 & 0.998 & 2.519 & 0.012 \\
0.950 & 0.525 & 0.997 & 2.029 & 0.042 \\
0.958 & 0.575 & 0.997 & 2.170 & 0.030 \\
0.929 & 0.423 & 0.996 & 1.748 & 0.081 \\
0.800 & 0.309 & 0.973 & 1.240 & 0.215 \\
0.833 & 0.369 & 0.977 & 1.469 & 0.142 \\
0.917 & 0.378 & 0.995 & 1.623 & 0.105 \\
0.833 & 0.770 & 0.882 & 7.851 & 0.000 \\
0.950 & 0.525 & 0.997 & 2.029 & 0.042 \\
0.952 & 0.729 & 0.993 & 2.924 & 0.003 \\
0.950 & 0.525 & 0.997 & 2.029 & 0.042 \\
0.900 & 0.326 & 0.994 & 1.474 & 0.140 \\
0.958 & 0.575 & 0.997 & 2.170 & 0.030 \\
0.900 & 0.533 & 0.986 & 2.084 & 0.037 \\
0.905 & 0.689 & 0.976 & 3.028 & 0.002 \\
0.928 & 0.849 & 0.968 & 6.029 & 0.000 \\
0.857 & 0.806 & 0.896 & 9.690 & 0.000 \\
& & & &
\end{tabular}

FIG. 4. The result of the meta-analysis and the meta-regression analysis, stratified by the use of cages for anterior fusion.

rate than the anterior fusion only group $(98.8 \%$ vs $86.4 \%$, p $<0.001)$. Lastly, none of the specific graft options analyzed here, including structural ICABG, structural allograft, rib structural autograft, vascularized autograft, or cage filled with bone graft or bone graft substitute/extender, demonstrated better or worse fusion outcomes with statistical significance. In multivariate analyses, tumors in the lumbar spine and/or sacrum were significantly associated with a

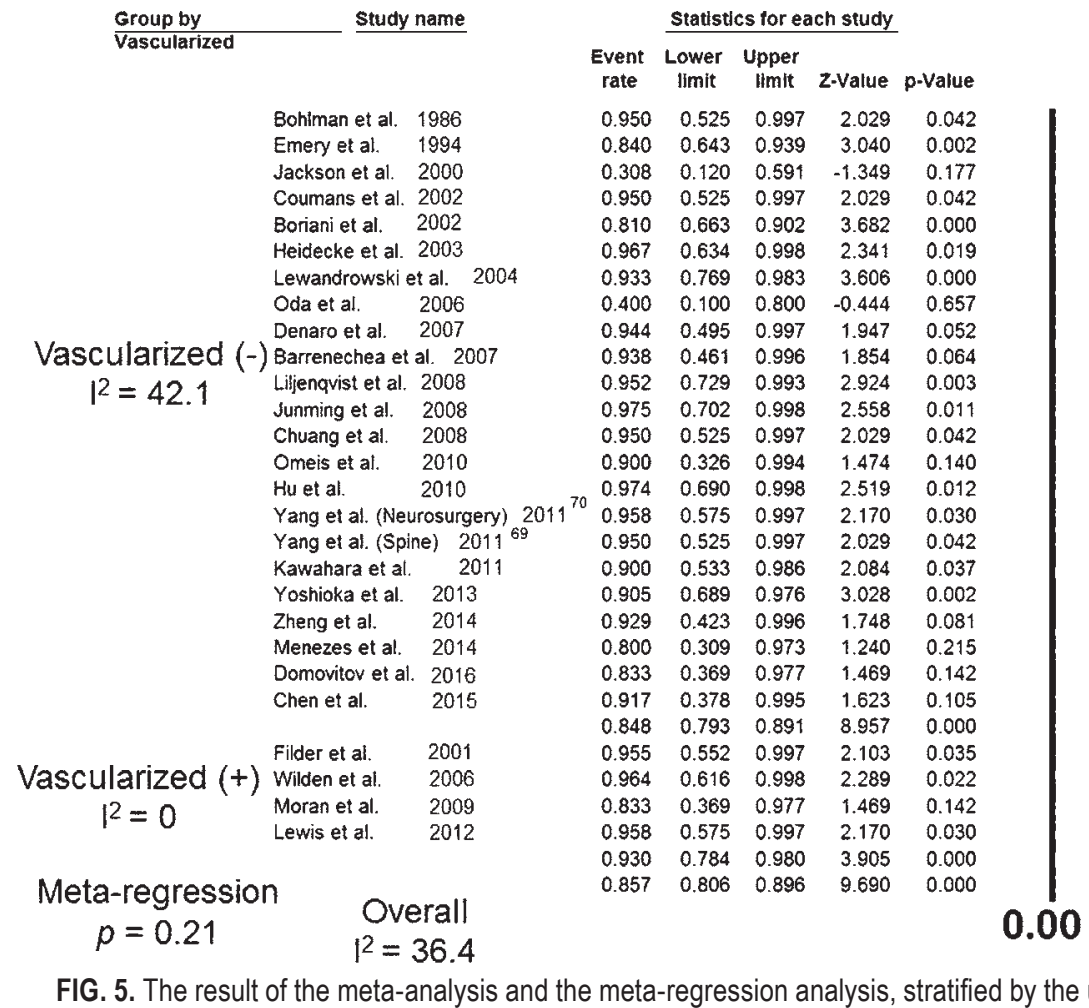

Group by

Coumans et al. 2002

Oda et al. $\quad 2006$

Vascularized $(-)$

Hu et al

Yang et al. (Neurosurgery) 2011

2013

Zheng et al. 2014

Menezes et al. 2014

Domovitov et al. 2016

Filder et al. $\quad 200$

Vascularized
\[ 2=0 \]

(1)

eta-regression

$$
p=0.21 \quad 1^{2}=36.4
$$

Statistics for each study

Event Lower Upper

rate limit limit z-Value o-Value

$\begin{array}{llllll}0.308 & 0.120 & 0.594 & -1.349 & 0.177\end{array}$

$\begin{array}{llllll}0.950 & 0.525 & 0.997 & 2.029 & 0.042\end{array}$

$\begin{array}{lllll}0.967 & 0.663 & 0.902 & 3.682 & 0.000\end{array}$

$\begin{array}{lllll}0.400 & 0.100 & 0.800 & -0.444 & 0.657\end{array}$

$\begin{array}{llllll}0.944 & 0.495 & 0.997 & 1.947 & 0.052\end{array}$

$\begin{array}{llllll}0.938 & 0.461 & 0.996 & 1.854 & 0.064\end{array}$

$\begin{array}{llllll}0.952 & 0.729 & 0.993 & 2.924 & 0.003\end{array}$

$\begin{array}{llllll}0.975 & 0.702 & 0.998 & 2.558 & 0.011\end{array}$

$\begin{array}{lllll}0.950 & 0.525 & 0.997 & 2.029 & 0.042\end{array}$

$\begin{array}{lllllllllll}0.900 & 0.326 & 0.994 & 1.474 & 0.140\end{array}$

$\begin{array}{llllll}0.958 & 0.575 & 0.997 & 2.170 & 0.030\end{array}$

$\begin{array}{llllll}0.950 & 0.525 & 0.997 & 2.029 & 0.042\end{array}$

$\begin{array}{lllll}0.905 & 0.689 & 0.976 & 3.084 & 0.037\end{array}$

$\begin{array}{llllll}0.929 & 0.423 & 0.996 & 1.748 & 0.081\end{array}$

$\begin{array}{llllll}0.800 & 0.309 & 0.973 & 1.240 & 0.215\end{array}$

$\begin{array}{llllll}0.833 & 0.369 & 0.977 & 1.469 & 0.14\end{array}$

$\begin{array}{lllll}0.848 & 0.378 & 0.995 & 1.623 & 0.105\end{array}$

$\begin{array}{llllll}0.055 & 0.552 & 0.097 & 2.103 & 0.035\end{array}$

$\begin{array}{lllll}0.964 & 0.616 & 0.998 & 2.289 & 0.022\end{array}$

$\begin{array}{lllll}0.958 & 0.369 & 0.977 & 1.469 & 0.142 \\ 0.997 & 2.170 & 0.030\end{array}$

$\begin{array}{lllll}0.930 & 0.784 & 0.980 & 3.905 & 0.000\end{array}$

$\begin{array}{lllll}0.857 & 0.806 & 0.896 & 9.690 & 0.000\end{array}$
Event rate and $95 \% \mathrm{Cl}$

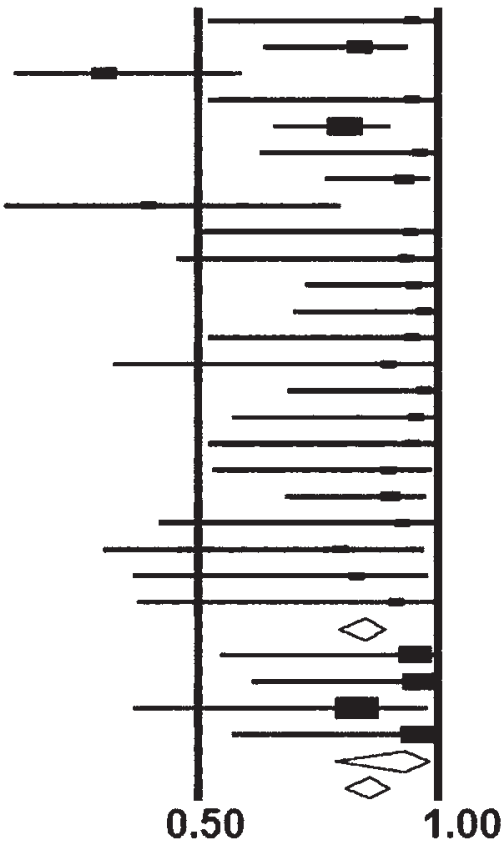

FIG. 5. The result of the meta-analysis and the meta-regression analysis, stratified by the use of vascularized autografts. 


\begin{tabular}{|c|c|c|}
\hline \multicolumn{2}{|l|}{$\begin{array}{ll}\text { Group by } \\
\text { fusion primary }\end{array}$} & Study name \\
\hline & $\begin{array}{l}\text { Bohlman et al. } \\
\text { Filder et al. } \\
\text { Coumans et al. } \\
\text { Heidecke et al. } \\
\text { Oda et al. } \\
\text { Barrenechea et al }\end{array}$ & $\begin{array}{l}1986 \\
2001 \\
2002 \\
2003 \\
2006 \\
\text { al. } 2007\end{array}$ \\
\hline \multirow{2}{*}{$\begin{array}{l}\text { Fusion assessment is } \\
\text { not primary outcome }\end{array}$} & Liljenquist et al. & 2008 \\
\hline & $\begin{array}{l}\text { Jurming et al. } \\
\text { Chuang et al. }\end{array}$ & $\begin{array}{l}2008 \\
2008\end{array}$ \\
\hline \multirow[t]{7}{*}{$R^{2}=0$} & $\begin{array}{l}\text { Hu et al. } \\
\text { Yang et al. (Neurc } \\
\text { Yang et al. (Spine }\end{array}$ & $\begin{array}{l}2010 \\
\text { rosurgery) } 2011^{70} \\
\text { le) } 20111^{70}\end{array}$ \\
\hline & Kawahara et al. & 2011 \\
\hline & Zheng et al. & 2014 \\
\hline & Domovitov et al. & 2016 \\
\hline & Chen et al. & 2015 \\
\hline & Emery et al. & 1994 \\
\hline & Jackson et al. & 2000 \\
\hline \multirow{3}{*}{$\begin{array}{c}\text { Fusion assessment is } \\
\text { primary outcome }\end{array}$} & Boriani et al. & 2002 \\
\hline & $\begin{array}{l}\text { Lewandrowski et } \\
\text { Wilden et al. }\end{array}$ & $\begin{array}{l}\text { tal. } 2004 \\
2006\end{array}$ \\
\hline & $\begin{array}{l}\text { Denaro et al. } \\
\text { Moran et al. }\end{array}$ & $\begin{array}{l}2007 \\
2009\end{array}$ \\
\hline \multirow{4}{*}{$P^{2}=55.1$} & Omeis et al. & 2010 \\
\hline & Lewis et al. & 2012 \\
\hline & Yoshioka et al. & 2013 \\
\hline & Menezes et al. & 2014 \\
\hline $\begin{array}{l}\text { Meta-regression } \\
\qquad p=0.02\end{array}$ & & $\begin{array}{l}\text { Dverall } \\
=36.4\end{array}$ \\
\hline
\end{tabular}

\begin{tabular}{|c|c|c|c|c|}
\hline \multirow[b]{2}{*}{$\begin{array}{c}\text { Event } \\
\text { rate }\end{array}$} & \multicolumn{4}{|c|}{ tistics io } \\
\hline & $\begin{array}{l}\text { Lower } \\
\text { limit }\end{array}$ & $\begin{array}{c}\text { Upper } \\
\text { limit }\end{array}$ & Value & p-valu \\
\hline 0.950 & 0.525 & 0.997 & 2.029 & \\
\hline 065 & & & & \\
\hline 1050 & 5 & & & \\
\hline 967 & 34 & & & \\
\hline 400 & 100 & & & \\
\hline 0.93 & & & & \\
\hline & & & & \\
\hline & & & & \\
\hline 0.950 & & & & \\
\hline & & & & \\
\hline & & & & \\
\hline 0.950 & 25 & & & \\
\hline & & & & \\
\hline & & & & \\
\hline & & & 169 & \\
\hline & & & & \\
\hline & & & & \\
\hline & & & & \\
\hline & & & & \\
\hline & & & & \\
\hline & & & & \\
\hline & & & & \\
\hline & & & & \\
\hline & & & & \\
\hline & & & & \\
\hline & & & & \\
\hline & & & & \\
\hline & & & & \\
\hline & & & & \\
\hline & & & & \\
\hline
\end{tabular}

FIG. 6. The result of the meta-analysis and the meta-regression analysis, stratified by primary outcomes.

lower fusion rate (OR 0.12, 95\% CI 0.02-0.74, $\mathrm{p}=0.02)$, whereas perioperative radiation trended toward a worse fusion outcome (OR 0.19, 95\% CI 0.02-1.68, p = 0.10).

\section{Time to Fusion Analysis}

Time to fusion was reported for 58 patients with an overall mean time to fusion of $5.4 \pm 1.4$ months (range 3-9 months), whereas 16 of 272 patients died before the confirmation of solid fusion with a mean survival of $3.1 \pm$ 2.1 months (range 0.5-6 months). The mean time to fusion was significantly longer in patients harboring metastatic tumors (6.0 vs 5.1 months, $\mathrm{p}=0.01$ ), patients who underwent perioperative radiation therapy (6.1 vs 4.3 months, $p$ $<0.001$ ), patients who received perioperative chemotherapy (6.0 vs 4.3 months, $p=0.02$ ), and patients who did not undergo preoperative embolization of tumors (5.9 vs 3.9 months, $\mathrm{p}<0.001$; Table 4). Additionally, Kaplan-Meier analysis revealed that vascularized grafts were associated with earlier fusion, compared with other graft options ( $\mathrm{p}=$ 0.03 ), with an HR of 5.2 (95\% CI 1.2-22.6; Fig. 7).

\section{Publication Bias}

The funnel plot is shown in Fig. 8, where the logit event rate $=\log$ (fusion rate/[1 - fusion rate $]$ ). A potential publication bias was suggested, with the $\mathrm{p}$ value of the classic fail-safe $\mathrm{N}$ test calculated to be 0.002 .

\section{Discussion}

To the best of our knowledge, this is the first systematic review and meta-analysis of bone graft options for spinal fusion following resection of spinal column tumors. The objective of this study was to review and meta-analyze the current literature and provide some insight into arthrod- esis outcomes in spinal oncology surgery. With a growing understanding of the biology of spinal tumors and the advent of novel adjunctive treatment options and reconstruction techniques, the overall survival of patients with spinal column tumors has been increasing. ${ }^{28,29,37}$ Thus, in addition to survival outcomes, other clinical considerations such as fusion outcomes, neurological outcomes, and pain outcomes should be taken into consideration to improve quality of life of the patients. Achieving solid arthrodesis is an important factor in the setting of resection of spinal column tumors, given the potentially long overall survival with likely instrumentation failure in the absence of arthrodesis. To achieve fusion, a wide variety of bone grafts, bone graft substitutes, and bone graft extenders have been explored in combination with instrumentation such as cages and pedicle screw/rod constructs. ${ }^{7,8,10,14,19,21,22,25,27,31,34,35}$, 38-42,45,48,54,55,67,69-71,74 However, because the majority of previous studies have been case reports, technical notes, or retrospective studies with small numbers of patients, it remains challenging to determine which patient populations benefit most from achieving fusion and what types of bone grafts should be used to maximize fusion rates.

The most significant limitation of this study was that the fusion rates reported in the reviewed literature varied widely from $36 \%$ to $100 \%$, which may be explained by the fact that articles included in the review were moderately heterogeneous. For instance, 14 articles included patients with both primary tumors and malignant tumors (intrastudy heterogeneity); the number of articles that included patients who underwent anterior fusion only, posterior fusion only, and miscellaneous, were 13,2 , and 12 , respectively (intra- and interstudy heterogeneity). Graft options used in each study varied widely within and between studies (intra- and interstudy heterogeneity), and the details on 
TABLE 3. Fusion rates stratified by each variable

\begin{tabular}{|c|c|c|c|c|c|c|c|}
\hline \multirow[b]{2}{*}{ Variable } & \multicolumn{4}{|c|}{ Univariate Analysis } & \multicolumn{3}{|c|}{ Multivariate Analysis } \\
\hline & Fusion $(n=250)$ & Nonfusion $(n=22)$ & Fusion rate (\%) & $p$ Value & OR & $95 \% \mathrm{Cl}$ & $p$ Value \\
\hline \multicolumn{8}{|l|}{ Baseline characteristics } \\
\hline Mean age $\pm S D$ (yrs) & $40.9 \pm 18.0$ & $32.7 \pm 18.7$ & & 0.18 & & & \\
\hline Mean tumor levels \pm SD & $1.8 \pm 1.1$ & $1.8 \pm 1.0$ & & 0.96 & & & \\
\hline Mean FU period \pm SD (mos) & $44.6 \pm 36.4$ & $56.6 \pm 82.6$ & & 0.28 & & & \\
\hline \multicolumn{8}{|l|}{ Tumor characteristics } \\
\hline Primary & 138 & 9 & 93.9 & 0.46 & & & \\
\hline Metastatic & 91 & 9 & 91.0 & & & & \\
\hline Benign & 90 & 4 & 95.7 & 0.21 & & & \\
\hline Malignant & 139 & 14 & 90.8 & & & & \\
\hline Osteolytic (+) & 43 & 7 & 86.0 & 0.32 & & & \\
\hline Osteolytic (-) & 46 & 3 & 93.9 & & & & \\
\hline Junction (+) & 60 & 11 & 84.5 & 0.02 & 0.62 & $(0.05-7.40)$ & 0.71 \\
\hline Junction (-) & 177 & 11 & 94.1 & & & & \\
\hline Lumbar and/or sacrum & 53 & 18 & 74.6 & $<0.001$ & 0.12 & $(0.02-0.74)$ & 0.02 \\
\hline Others & 184 & 4 & 97.9 & & & & \\
\hline \multicolumn{8}{|l|}{ Adjunctive treatment } \\
\hline $\mathrm{RT}(+)$ & 93 & 10 & 90.3 & 0.03 & 0.19 & $(0.02-1.68)$ & 0.10 \\
\hline $\mathrm{RT}(-)$ & 70 & 1 & 98.6 & & & & \\
\hline Chemo (+) & 14 & 0 & 100.0 & 1 & & & \\
\hline Chemo (-) & 85 & 4 & 95.5 & & & & \\
\hline Embo (+) & 23 & 3 & 88.5 & 0.18 & & & \\
\hline Embo (-) & 55 & 2 & 96.5 & & & & \\
\hline \multicolumn{8}{|l|}{ Graft } \\
\hline Anterior \& pst fusion & 85 & 1 & 98.8 & $<0.001$ & 0.42 & $(0.04-4.21)$ & 0.46 \\
\hline Anterior fusion only & 127 & 20 & 86.4 & & & & \\
\hline Structural ICABG (+) & 75 & 5 & 93.8 & 0.63 & & & \\
\hline Structural ICABG (-) & 175 & 17 & 91.1 & & & & \\
\hline Structural allograft (+) & 39 & 3 & 92.9 & 1 & & & \\
\hline Structural allograft (-) & 211 & 19 & 91.7 & & & & \\
\hline Rib structural autograft (+) & 30 & 1 & 96.8 & 0.49 & & & \\
\hline Rib structural autograft (-) & 220 & 21 & 91.3 & & & & \\
\hline Vascularized autograft (+) & 23 & 1 & 95.8 & 0.70 & & & \\
\hline Vascularized autograft (-) & 227 & 21 & 91.5 & & & & \\
\hline Cage $(+)$ & 74 & 3 & 96.1 & 0.14 & & & \\
\hline Cage (-) & 176 & 19 & 90.3 & & & & \\
\hline
\end{tabular}

$(+)=$ positive; $(-)=$ negative.

Boldface type indicates statistical significance.

adjunctive treatments were suboptimally reported across 27 studies (intra- and interstudy heterogeneity). Variables with interstudy heterogeneity, such as cage and vascularized graft status, were further analyzed with a meta-regression model, which revealed that the use of cages filled with morselized ICABG and/or other bone graft options was significantly associated with a higher fusion rate. This result suggests a potential advantage to reconstruction of the anterior column with a cage to provide early structural support, although the result may be biased as tumors are more likely to involve the vertebral body. On the other hand, vascularized bone graft did not demonstrate better fusion outcomes, presumably due to the limited number of studies.

Variables with intrastudy heterogeneity prevented further meta-analyses or meta-regression analyses, which warranted subsequent per-patient analyses. In per-patient analyses, the location of spinal column tumors in the lumbosacral spine was the only independent factor related to pseudarthrosis, which is compatible with nontumor studies and potentially due to the higher biomechanical loads in those areas. While perioperative RT demonstrated a trend toward a lower fusion rate, none of the modifiable factors, namely, graft and reconstruction options, were associated 
TABLE 4. Time to fusion stratified by each variable

\begin{tabular}{|c|c|c|c|}
\hline Variable & $\begin{array}{l}\text { No. of } \\
\text { Patients }\end{array}$ & $\begin{array}{c}\text { Mean Time to } \\
\text { Fusion } \pm \text { SD (mos) }\end{array}$ & $\begin{array}{c}p \\
\text { Value }\end{array}$ \\
\hline \multicolumn{4}{|l|}{ Patient characteristics } \\
\hline Male & 19 & $4.7 \pm 1.8$ & 0.47 \\
\hline Female & 9 & $5.2 \pm 1.9$ & \\
\hline \multicolumn{4}{|l|}{ Tumor characteristics } \\
\hline Primary & 33 & $5.1 \pm 1.7$ & 0.01 \\
\hline Metastatic & 25 & $6.0 \pm 0.2$ & \\
\hline Junction (+) & 7 & $5.4 \pm 2.6$ & 0.97 \\
\hline Junction (-) & 51 & $5.5 \pm 1.2$ & \\
\hline Lumbosacral & 21 & $5.5 \pm 1.1$ & 0.91 \\
\hline Others & 37 & $5.4 \pm 1.5$ & \\
\hline \multicolumn{4}{|l|}{ Adjunctive treatment } \\
\hline $\mathrm{RT}(+)$ & 39 & $6.1 \pm 0.9$ & $<0.001$ \\
\hline RT (-) & 16 & $4.3 \pm 1.4$ & \\
\hline Chemo $(+)$ & 5 & $6.0 \pm 0.0$ & 0.02 \\
\hline Chemo (-) & 16 & $4.3 \pm 1.4$ & \\
\hline Embo $(+)$ & 12 & $3.9 \pm 3.8$ & $<0.001$ \\
\hline Embo (-) & 39 & $5.9 \pm 0.3$ & \\
\hline \multicolumn{4}{|l|}{ Graft } \\
\hline ICABG (+) & 4 & $6.0 \pm 3.5$ & 0.41 \\
\hline ICABG (-) & 54 & $5.4 \pm 1.1$ & \\
\hline Rib autograft (+) & 13 & $5.8 \pm 0.6$ & 0.34 \\
\hline Rib autograft (-) & 45 & $5.4 \pm 1.5$ & \\
\hline Vacularized autograft (+) & 9 & $4.8 \pm 1.3$ & 0.09 \\
\hline Vacularized autograft (-) & 49 & $5.6 \pm 1.0$ & \\
\hline
\end{tabular}

Boldface type indicates statistical significance or trend toward significance.

with fusion outcomes in multivariate analysis. However, if meta-regression and univariate analysis of this study were taken into consideration, the use of cages filled with morselized ICABG and/or other bone graft options, and combined anterior and posterior fusion, were associated with better fusion outcomes. In terms of bone graft options for posterior fusion, only 2 studies reported posterior-only fusion in 23 patients, which made it impossible to separately analyze the data.

BMP-2 was not used in any of the patients included in this study, which indicated that there remains significant concern regarding potential carcinogenicity and contraindications in oncology patients. While a recent meta-analysis by Cahill et al. ${ }^{11}$ revealed that there was no conclusive evidence that BMP-2 was associated with the formation of cancer locally or at a distant site, there remains concern regarding its potential carcinogenicity. ${ }^{58,62,63,66}$

Although the use of local bone grafts are relatively contraindicated following spinal column tumor resection, patients in 3 studies ${ }^{19,41,54}$ received morselized local bone grafts. Of note, Coumans et al. ${ }^{19}$ reported the use of a vertebral autograft that contained tumor, after the diagnosis of benign intraosseous schwannoma was confirmed by intraoperative pathological diagnosis. Also, it has been demonstrated that following spondylectomy, vertebral bodies with tumors could be used as morselized bone grafts in

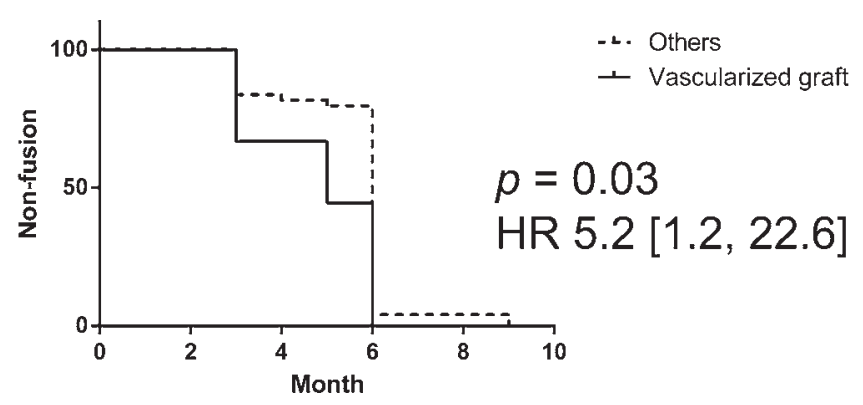

FIG. 7. Kaplan-Meier curves on time to fusion, stratified by the use of vascularized autografts.

conjunction with cages if they were placed into liquid nitrogen $\left(-196^{\circ} \mathrm{C}\right)$ for 20 minutes. ${ }^{49-52,61}$ Surprisingly, this technique was applied to malignant tumors as well, and with an average follow-up of 14 months, none of the 56 patients (49 with metastatic tumors) developed recurrence in the grafted bone inside cages ${ }^{51}$ It was also demonstrated that the implantation of a tumor-bearing graft could systemically induce antitumor immune response in human studies. ${ }^{49,50}$ Future multicenter, prospective studies regarding these intriguing treatments with longer follow-up periods would be necessary to better elucidate their safety features and true advantages. This surgical strategy remains controversial, considering the possibility of tumor recurrence or malignant transformation of some tumor histologies.

There are advantages to using vascularized autografts, either from ribs or fibulas, which merit further discussion, although these should be weighed against the more invasive surgical procedure. In this analysis, time to fusion was discovered to be shorter in patients receiving vascularized autografts, but the fusion rate itself did not differ significantly from the other bone graft options. This is partly because the number of patients was small in this group but also potentially because $57 \%$ to $77 \%$ of patients with vascularized autografts received perioperative RT. However, it should be considered that complication rates for this procedure are relatively high, and should be weighed against the potential benefit. ${ }^{1,27,36,41,48,67}$ For instance, Minami et al. ${ }^{47}$ reported that the procedure had to be abandoned in 3 of 16 patients because of difficulties with microsurgical anastomosis. Also, the mean \pm standard deviation time needed for the harvest, rib preparation, and anastomosis was $58 \pm 13$ minutes. ${ }^{51}$ To the best of knowledge, there are no studies comparing morbidities associated with surgical technique between ICABG,${ }^{12}$ fibula autograft,${ }^{65}$ and vascularized autografts. Considering the significant morbidity, vascularized grafting procedures should not be recommended for patients with limited life expectancy. In summary, there is not overwhelming data to support the use of vascularized autografts, although they are likely beneficial in particularly challenging fusion environments in which survival greater than 12 months is expected, and future prospective studies should be performed.

Regarding other factors related to fusion outcomes, it was found that adjunctive treatment was significantly associated with fusion rates as well as time to fusion, which is similar to the results of previous studies. For instance, Orgel et al ${ }^{56}$ reported that patients receiving chemotherapy 


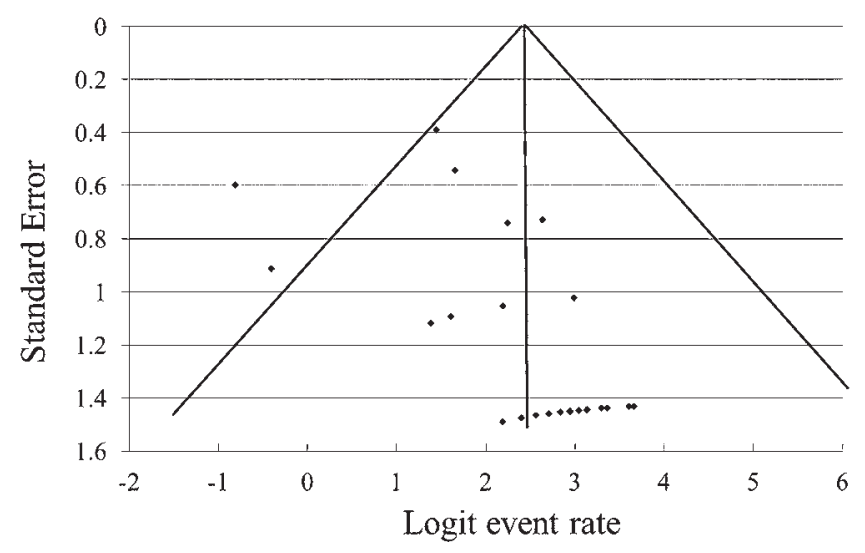

FIG. 8. Funnel plot of standard error by logit event rate, where the logit event rate $=\log$ (fusion rate/[1 - fusion rate $]$ ).

for acute lymphoblastic leukemia had substantial injury to corticocancellous bones. Additionally, the impact of RT and PBT $^{3,23,25,43,44}$ on BMD and fusion outcomes has been previously described. Interestingly, denosumab is currently used as treatment for giant cell tumors as well as osteoporosis..$^{20,72}$ Long-term follow-up of patients who underwent postoperative PBT with or without denosumab might be warranted to clarify a supplemental benefit of this newly developed molecular-targeted therapy. Additionally, the potential influence of preoperative embolization on time to fusion was observed in this study. It can be hypothesized that reduced bleeding from the surgical cavity contributed to less dissection and less coagulation of surrounding tissues, which in turn, resulted in a more vascularized fusion bed to promote formation of fusion mass. Alternatively, the hypoxic preconditioning of mesenchymal stem cells in the fusion bed can induce neo-vascularization in an early stage, as several in vitro studies have previously demonstrated..$^{6,26}$

Lastly, intention to achieve arthrodesis based on expected survival merits further discussion. For instance, Coumans et al. ${ }^{19}$ advocated 6 months as a cutoff value for the indication of fusion, while Oda et al. ${ }^{54}$ proposed 12 months. However, others stated that factors related to spinal instability such as lumbosacral involvement, ${ }^{48}$ or more than 3-level spondylectomies, ${ }^{71}$ should be considered. Clearly, this decision is patient-specific, but should be considered further given increasing survival times with potential for hardware failure in the absence of arthrodesis. In our systematic review, an ad-hoc analysis revealed that 16 of 272 patients died before the confirmation of solid fusion with a mean survival of $3.1 \pm 2.1$ months (range $0.5-6$ months). In contrast, the mean time to fusion was $5.4 \pm 1.4$ months (range 3-9 months). Considering these results, we recommend that in patients with estimated life expectancy less than 6-12 months, such as those with a Tokuhashi score $<8$ (i.e., harboring an unresectable lung cancer with multiple extraspinal and spinal bone metastases), instrumented stabilization with no attempted arthrodesis would be most reasonable. However, in patients with breast metastases with minimal systemic tumor burden, life expectancy often exceeds 1 year, ${ }^{13,60}$ and an attempted arthrodesis should be strongly considered. Additionally, in patients with primary tumors in whom an en bloc resection can be performed suc- cessfully, there is often greater longevity; therefore, an attempted arthrodesis should be performed, as the hardware will eventually fail in the absence of fusion. To further clarify this issue, it would be interesting to investigate other studies in which cages without any bone graft were used for reconstruction to determine how long instrumentation itself can last without causing significant complications such as device subsidence, ${ }^{4,68}$ device fractures, and kyphosis in adjacent levels, in the oncological patient population.

The general limitations of meta-analyses can be applied to this meta-analysis as well, such as the influences of bias that existed in each study on the results of the meta-analysis. Furthermore, as shown in Fig. 8, a publication bias existed in this meta-analysis, which was mainly due to negative publication bias, i.e., articles in which fusion rates were suboptimal (less than PFR of $85.7 \%$ ) might have been missing from the included studies. In addition, heterogeneity among patient, disease, surgical, and study characteristics significantly limits drawing strong conclusions from our study. Moreover, as discussed above, some of the missing data on materials and methods in certain articles, particularly relevant variables such as perioperative chemotherapy, RT, and preoperative embolization, biased the results. It is notable that the studies in which arthrodesis was assessed as a primary outcome showed a significantly lower fusion rate than the others, because their fusion criteria were usually more stringent. For instance, Denaro et al. ${ }^{21}$ used strict motion criteria along with an assessment of bridging bone, while Zheng et al. ${ }^{74}$ ambiguously defined the follow-up strategy based on plain radiographs without any specific fusion criteria. Therefore, to further optimize the quality of each clinical study involving spinal column tumors in the future, authors should more specifically report fusion assessments and fusion outcomes.

\section{Conclusions}

To the best of our knowledge, this is the first systematic review and meta-analysis of bone graft options for spinal fusion following resection of spinal column tumors. Overall, fusion rates varied from $36.0 \%$ to $100.0 \%$ due to both inter- and intrastudy heterogeneity, with a PFR of $85.7 \%$. Due to overall differences in patient, disease, fusion criteria, and treatment characteristics, the optimal surgical techniques and factors predictive of fusion remain unclear. In terms of the intention to achieve arthrodesis, patients with an estimated life expectancy less than 6-12 months do not necessarily require fusion procedures, whereas in patients expected to live longer than 1 year, attempted arthrodesis should be strongly considered in addition to factors related to procedural morbidity. Because the impact of RT, chemotherapy, embolization, and fusion assessment on fusion outcomes was significant, it is highly recommended that in future studies, each relevant variable-such as tumor histology, tumor location, adjunctive treatment, bone graft, fusion criteria, and fusion outcomes - should be appropriately investigated.

\section{Acknowledgments}

We would like to acknowledge research support from the Gordon and Marilyn Macklin Foundation. 


\section{References}

1. Ackerman DB, Rose PS, Moran SL, Dekutoski MB, Bishop AT, Shin AY: The results of vascularized-free fibular grafts in complex spinal reconstruction. J Spinal Disord Tech 24:170-176, 2011

2. Alfieri A, Gazzeri R, Neroni M, Fiore C, Galarza M, Esposito S: Anterior expandable cylindrical cage reconstruction after cervical spinal metastasis resection. Clin Neurol Neurosurg 113:914-917, 2011

3. Amichetti M, Cianchetti M, Amelio D, Enrici RM, Minniti G: Proton therapy in chordoma of the base of the skull: a systematic review. Neurosurg Rev 32:403-416, 2009

4. Arbit E, Galicich JH: Vertebral body reconstruction with a modified Harrington rod distraction system for stabilization of the spine affected with metastatic disease. J Neurosurg 83:617-620, 1995

5. Arıkan M, Togral G, Hasturk AE, Kekec F, Parpucu $\mathrm{M}$, Gungor S: Management of sacral tumors requiring spino-pelvic reconstruction with different histopathologic diagnosis: evaluation with four cases. Asian Spine J 9:971977,2015

6. Bader AM, Klose K, Bieback K, Korinth D, Schneider M, Seifert M, et al: Hypoxic preconditioning increases survival and pro-angiogenic capacity of human cord blood mesenchymal stromal cells in vitro. PLoS One 10:e0138477, 2015

7. Barrenechea IJ, Perin NI, Triana A, Lesser J, Costantino P, Sen C: Surgical management of chordomas of the cervical spine. J Neurosurg Spine 6:398-406, 2007

8. Bohlman HH, Sachs BL, Carter JR, Riley L, Robinson RA: Primary neoplasms of the cervical spine. Diagnosis and treatment of twenty-three patients. J Bone Joint Surg Am 68:483-494, 1986

9. Boriani S, Biagini R, Bandiera S, Gasbarrini A, De Iure F: Reconstruction of the anterior column of the thoracic and lumbar spine with a carbon fiber stackable cage system. Orthopedics 25:37-42, 2002

10. Boriani S, Biagini R, De Iure F, Bertoni F, Malaguti MC, Di Fiore M, et al: En bloc resections of bone tumors of the thoracolumbar spine. A preliminary report on 29 patients. Spine (Phila Pa 1976) 21:1927-1931, 1996

11. Cahill KS, McCormick PC, Levi AD: A comprehensive assessment of the risk of bone morphogenetic protein use in spinal fusion surgery and postoperative cancer diagnosis. J Neurosurg Spine 23:86-93, 2015

12. Calori GM, Colombo M, Mazza EL, Mazzola S, Malagoli E, Mineo GV: Incidence of donor site morbidity following harvesting from iliac crest or RIA graft. Injury 45 (Suppl 6):S116-S120, 2014

13. Chaichana KL, Pendleton C, Sciubba DM, Wolinsky JP, Gokaslan ZL: Outcome following decompressive surgery for different histological types of metastatic tumors causing epidural spinal cord compression. Clinical article. J Neurosurg Spine 11:56-63, 2009

14. Chen G, Li J, Li X, Fan H, Guo Z, Wang Z: Giant cell tumor of axial vertebra: surgical experience of five cases and a review of the literature. World J Surg Oncol 13:62, 2015

15. Chong S, Shin SH, Yoo H, Lee SH, Kim KJ, Jahng TA, et al: Single-stage posterior decompression and stabilization for metastasis of the thoracic spine: prognostic factors for functional outcome and patients' survival. Spine J 12:1083-1092, 2012

16. Chuang HC, Wei ST, Lee HC, Chen CC, Lee WY, Cho DY: Preliminary experience of titanium mesh cages for pathological fracture of middle and lower cervical vertebrae. J Clin Neurosci 15:1210-1215, 2008

17. Clarke MJ, Mendel E, Vrionis FD: Primary spine tumors: diagnosis and treatment. Cancer Contr 21:114-123, 2014

18. Colman MW, Karim SM, Lozano-Calderon SA, Pedlow FX, Raskin KA, Hornicek FJ, et al: Quality of life after en bloc resection of tumors in the mobile spine. Spine J 15:17281737,2015

19. Coumans JV, Marchek CP, Henderson FC: Use of the telescopic plate spacer in treatment of cervical and cervicothoracic spine tumors. Neurosurgery 51:417-426, 2002

20. de Carvalho Cavalcante RA, Silva Marques RA, dos Santos VG, Sabino E, Fraga AC Jr, Zaccariotti VA, et al: Spondylectomy for giant cell tumor after denosumab therapy. Spine (Phila Pa 1976) 41:E178-E182, 2016

21. Denaro V, Denaro L, Papalia R, Marinozzi A, Di Martino A: Surgical management of cervical spine osteoblastomas. Clin Orthop Relat Res 455:190-195, 2007

22. Domovitov SV, Chandhanayingyong C, Boland PJ, McKeown DG, Healey JH: Conservative surgery in the treatment of giant cell tumor of the sacrum: 35 years' experience. J Neurosurg Spine 24:228-240, 2016

23. Doyen J, Falk AT, Floquet V, Hérault J, Hannoun-Lévi JM: Proton beams in cancer treatments: clinical outcomes and dosimetric comparisons with photon therapy. Cancer Treat Rev 43:104-112, 2016

24. Elder BD, Sankey EW, Goodwin CR, Kosztowski TA, Lo SF, Bydon A, et al: Surgical outcomes in patients with high spinal instability neoplasm score secondary to spinal giant cell tumors. Global Spine J 6:21-28, 2016

25. Emery SE, Hughes SS, Junglas WA, Herrington SJ, Pathria $\mathrm{MN}$ : The fate of anterior vertebral bone grafts in patients irradiated for neoplasm. Clin Orthop Relat Res (300):207212, 1994

26. Fan L, Zhang C, Yu Z, Shi Z, Dang X, Wang K: Transplantation of hypoxia preconditioned bone marrow mesenchymal stem cells enhances angiogenesis and osteogenesis in rabbit femoral head osteonecrosis. Bone 81:544-553, 2015

27. Fidler MW: Surgical treatment of giant cell tumours of the thoracic and lumbar spine: report of nine patients. Eur Spine J 10:69-77, 2001

28. Garofalo F, di Summa PG, Christoforidis D, Pracht M, Laudato P, Cherix S, et al: Multidisciplinary approach of lumbosacral chordoma: From oncological treatment to reconstructive surgery. J Surg Oncol 112:544-554, 2015

29. Greco C, Pares O, Pimentel N, Moser E, Louro V, Morales $\mathrm{X}$, et al: Spinal metastases: From conventional fractionated radiotherapy to single-dose SBRT. Rep Pract Oncol Radiother 20:454-463, 2015

30. Harel R, Chao S, Krishnaney A, Emch T, Benzel EC, Angelov L: Spine instrumentation failure after spine tumor resection and radiation: comparing conventional radiotherapy with stereotactic radiosurgery outcomes. World Neurosurg 74:517-522, 2010

31. Heidecke V, Rainov NG, Burkert W: Results and outcome of neurosurgical treatment for extradural metastases in the cervical spine. Acta Neurochir (Wien) 145:873-881, 2003

32. Higgins JPT, Thompson SG, Deeks JJ, Altman DG: Measuring inconsistency in meta-analyses. BMJ 327:557-560, 2003

33. Hobusch GM, Tiefenboeck TM, Patsch J, Krall C, Holzer G: Do patients after chondrosarcoma treatment have age-appropriate bone mineral density in the long term? Clin Orthop Relat Res 474:1508-1515, 2016

34. Hu Y, Xia Q, Ji J, Miao J: One-stage combined posterior and anterior approaches for excising thoracolumbar and lumbar tumors: surgical and oncological outcomes. Spine (Phila Pa 1976) 35:590-595, 2010

35. Jackson RJ, Gokaslan ZL: Spinal-pelvic fixation in patients with lumbosacral neoplasms. J Neurosurg 92 (1 Suppl): $61-70,2000$

36. Jandali S, Diluna ML, Storm PB, Low DW: Use of the vascularized free fibula graft with an arteriovenous loop for fusion of cervical and thoracic spinal defects in previously irradiated pediatric patients. Plast Reconstr Surg 127:1932-1938, 2011 
37. Joaquim AF, Powers A, Laufer I, Bilsky MH: An update in the management of spinal metastases. Arq Neuropsiquiatr 73:795-802, 2015

38. Junming M, Cheng Y, Dong C, Jianru X, Xinghai Y, Quan $\mathrm{H}$, et al: Giant cell tumor of the cervical spine: a series of 22 cases and outcomes. Spine (Phila Pa 1976) 33:280-288, 2008

39. Kawahara N, Tomita K, Murakami H, Demura S, Yoshioka K, Kato S: Total en bloc spondylectomy of the lower lumbar spine: a surgical techniques of combined posterior-anterior approach. Spine (Phila Pa 1976) 36:74-82, 2011

40. Lewandrowski KU, Hecht AC, DeLaney TF, Chapman PA, Hornicek FJ, Pedlow FX: Anterior spinal arthrodesis with structural cortical allografts and instrumentation for spine tumor surgery. Spine (Phila Pa 1976) 29:1150-1159, 2004

41. Lewis SJ, Kulkarni AG, Rampersaud YR, Jhaveri S, Quraishi $\mathrm{N}$, Bacon SA, et al: Posterior column reconstruction with autologous rib graft after en bloc tumor excision. Spine (Phila Pa 1976) 37:346-350, 2012

42. Liljenqvist $U$, Lerner T, Halm H, Buerger $H$, Gosheger G, Winkelmann W: En bloc spondylectomy in malignant tumors of the spine. Eur Spine J 17:600-609, 2008

43. Mahajan A: Normal tissue complications from low-dose proton therapy. Health Phys 103:586-589, 2012

44. Matsumoto M, Watanabe K, Tsuji T, Ishii K, Nakamura M, Chiba K, et al: Late instrumentation failure after total en bloc spondylectomy. J Neurosurg Spine 15:320-327, 2011

45. Menezes AH, Ahmed R: Primary atlantoaxial bone tumors in children: management strategies and long-term follow-up. J Neurosurg Pediatr 13:260-272, 2014

46. Metcalfe S, Gbejuade H, Patel NR: The posterior transpedicular approach for circumferential decompression and instrumented stabilization with titanium cage vertebrectomy reconstruction for spinal tumors: consecutive case series of 50 patients. Spine (Phila Pa 1976) 37:1375-1383, 2012

47. Minami A, Kaneda K, Satoh S, Abumi K, Kutsumi K: Free vascularised fibular strut graft for anterior spinal fusion. J Bone Joint Surg Br 79:43-47, 1997

48. Moran SL, Bakri K, Mardini S, Shin AY, Bishop AT: The use of vascularized fibular grafts for the reconstruction of spinal and sacral defects. Microsurgery 29:393-400, 2009

49. Murakami H, Demura S, Kato S, Nishida H, Yoshioka K, Hayashi $\mathrm{H}$, et al: Increase of IL-12 following reconstruction for total en bloc spondylectomy using frozen autografts treated with liquid nitrogen. PLoS One 8:e64818, 2013

50. Murakami H, Demura S, Kato S, Yoshioka K, Hayashi H, Inoue $\mathrm{K}$, et al: Systemic antitumor immune response following reconstruction using frozen autografts for total en bloc spondylectomy. Spine J 14:1567-1571, 2014

51. Murakami H, Kato S, Demura S, Yoshioka K, Hayashi H, Inoue K, et al: Novel reconstruction technique using a frozen tumor-bearing vertebra from a total en bloc sponydylectomy for spinal tumors. Orthopedics 36:605-607, 2013

52. Murakami H, Kato S, Ueda Y, Fujimaki Y, Tsuchiya H: Reconstruction using a frozen tumor-bearing vertebra in total en bloc spondylectomy can enhance antitumor immunity. Eur Spine J 23 (Suppl 2):222-227, 2014

53. Narayan P, Haid RW, Subach BR, Comey CH, Rodts GE: Effect of spinal disease on successful arthrodesis in lumbar pedicle screw fixation. J Neurosurg 97 (3 Suppl):277-280, 2002

54. Oda I, Abumi K, Ito M, Kotani Y, Oya T, Hasegawa K, et al: Palliative spinal reconstruction using cervical pedicle screws for metastatic lesions of the spine: a retrospective analysis of 32 cases. Spine (Phila Pa 1976) 31:1439-1444, 2006

55. Omeis I, Bekelis K, Gregory A, McGirt M, Sciubba D, Bydon A, et al: The use of expandable cages in patients undergoing multilevel corpectomies for metastatic tumors in the cervical spine. Orthopedics 33:87-92, 2010
56. Orgel E, Mueske NM, Wren TAL, Gilsanz V, Butturini AM, Freyer DR, et al: Early injury to cortical and cancellous bone from induction chemotherapy for adolescents and young adults treated for acute lymphoblastic leukemia. Bone 85:131-137, 2016

57. Orwin R: A fail-safe $\mathrm{N}$ for effect size in meta-analysis. J Educ Stat 8:157-159, 1983

58. Sayama C, Willsey M, Chintagumpala M, Brayton A, Briceño V, Ryan SL, et al: Routine use of recombinant human bone morphogenetic protein-2 in posterior fusions of the pediatric spine and incidence of cancer. J Neurosurg Pediatr 16:4-13, 2015

59. Shamseer L, Moher D, Clarke M, Ghersi D, Liberati A, Petticrew M, et al: Preferred reporting items for systematic review and meta-analysis protocols (PRISMA-P) 2015: elaboration and explanation. BMJ 349:g7647, 2015

60. Shehadi JA, Sciubba DM, Suk I, Suki D, Maldaun MV, McCutcheon IE, et al: Surgical treatment strategies and outcome in patients with breast cancer metastatic to the spine: a review of 87 patients. Eur Spine J 16:1179-1192, 2007

61. Shinmura K, Murakami H, Demura S, Kato S, Yoshioka K, Hayashi $\mathrm{H}$, et al: Implantation of liquid nitrogen frozen tumor tissue after posterior decompression and stabilization for metastatic spinal tumors. Asian Spine J 9:869-875, 2015

62. Skovrlj B, Koehler SM, Anderson PA, Qureshi SA, Hecht AC, Iatridis JC, et al: Association between BMP-2 and carcinogenicity. Spine (Phila Pa 1976) 40:1862-1871, 2015

63. Sonn KA, Kannan AS, Bellary SS, Yun C, Hashmi SZ, Nelson JT, et al: The effect of recombinant human bone morphogenetic protein- 2 on a novel lung cancer spine metastasis model in rodents. J Orthop Res 34:1274-1281, 2016

64. Tokuhashi Y, Uei H, Oshima M, Ajiro Y: Scoring system for prediction of metastatic spine tumor prognosis. World J Orthop 5:262-271, 2014

65. Vail TP, Urbaniak JR: Donor-site morbidity with use of vascularized autogenous fibular grafts. J Bone Joint Surg Am 78:204-211, 1996

66. Vavken J, Mameghani A, Vavken P, Schaeren S: Complications and cancer rates in spine fusion with recombinant human bone morphogenetic protein-2 (rhBMP-2). Eur Spine J [epub ahead of print], 2015

67. Wilden JA, Moran SL, Dekutoski MB, Bishop AT, Shin AYS: Results of vascularized rib grafts in complex spinal reconstruction. J Bone Joint Surg Am 88:832-839, 2006

68. Wu J, Luo D, Ye X, Luo X, Yan L, Qian H: Anatomy-related risk factors for the subsidence of titanium mesh cage in cervical reconstruction after one-level corpectomy. Int J Clin Exp Med 8:7405-7411, 2015

69. Yang X, Huang W, Xiao J, Wu Z, Feng D, Zheng W, et al: Combined pre- and retrovascular extraoral approach for tumors at lateral mass of the atlas. Spine (Phila Pa 1976) 36:129-136, 2011

70. Yang X, Wu Z, Xiao J, Teng H, Feng D, Huang W, et al: Sequentially staged resection and 2-column reconstruction for C2 tumors through a combined anterior retropharyngeal-posterior approach: surgical technique and results in 11 patients. Neurosurgery (2 Suppl Operative) 69:ons184-ons194, 2011

71. Yoshioka K, Murakami H, Demura S, Kato S, Kawahara N, Tomita K, et al: Clinical outcome of spinal reconstruction after total en bloc spondylectomy at 3 or more levels. Spine (Phila Pa 1976) 38:E1511-E1516, 2013

72. Zaheer S, LeBoff M, Lewiecki EM: Denosumab for the treatment of osteoporosis. Expert Opin Drug Metab Toxicol 11:461-470, 2015

73. Zaidi HA, Awad AW, Dickman CA: Complete spondylectomy using orthogonal spinal fixation and combined anterior and posterior approaches for thoracolumbar spinal reconstruction: technical nuances and clinical results. J Spinal Disord Tech [epub ahead of print], 2015 
74. Zheng W, Wu J, Wu Z, Xiao J: Atlantoaxial instability secondary to eosinophilic granuloma of the axis in adults: longterm follow-up in six cases. Spine J 14:2701-2709, 2014

\section{Disclosures}

Dr. Goodwin is a UNCF-Merck postdoctoral fellow and has received an award from the Burroughs Wellcome Fund. Dr. Bydon has received a research grant from DePuy Spine and serves on the clinical advisory board of MedImmune, LLC. Dr. Gokaslan has stock ownership in US Spine and Spinal Kinetics; has performed consulting, speaking, and teaching for the AO Foundation; and has received research support from DePuy, NREF, AOSpine, and AO North America. Dr. Sciubba has served as a consultant for Medtronic, Stryker, DePuy Synthes, Globus, and Orthofix. Dr. Witham has received non-study-related research support from the Gordon and Marilyn Macklin Foundation, and research materials from Eli Lilly and Co. This article reflects the views of the authors and should not be construed to represent the FDA's views or policies.

\section{Author Contributions}

Conception and design: Elder, Ishida, Goodwin, Sciubba, Wolinsky. Acquisition of data: Elder, Ishida. Analysis and interpretation of data: Elder, Ishida, Goodwin, Bydon, Gokaslan, Sciubba, Witham. Drafting the article: Elder, Ishida. Critically revising the article: Elder, Goodwin, Bydon, Gokaslan, Sciubba, Wolinsky, Witham. Reviewed submitted version of manuscript: all authors. Approved the final version of the manuscript on behalf of all authors: Elder. Statistical analysis: Ishida. Study supervision: Gokaslan, Sciubba, Wolinsky, Witham.

\section{Correspondence}

Benjamin Elder, Department of Neurosurgery, The Johns Hopkins University School of Medicine, 1800 Orleans St., Rm. 6007, Baltimore, MD 21287. email: belder4@jhmi.edu. 